\title{
Structural network alterations in focal and generalized epilepsy follow axes of epilepsy risk gene expression: An ENIGMA study
}

\begin{abstract}
Sara Larivière ${ }^{1,}$, Jessica Royer ${ }^{1}$, Raúl Rodríguez-Cruces ${ }^{1}$, Maria Eugenia Caligiuri ${ }^{2}$, Antonio Gambardella ${ }^{2,3}$, Luis Concha ${ }^{4}$, Simon S. Keller ${ }^{5,6}$, Fernando Cendes ${ }^{7}$, Clarissa L. Yasuda ${ }^{7}$, Leonardo Bonilha ${ }^{8}$, Ezequiel Gleichgerrcht ${ }^{8}$, Niels K. Focke ${ }^{9}$, Martin Domin ${ }^{10}$, Felix von Podewills ${ }^{11}$, Soenke Langner ${ }^{12}$, Christian Rummel ${ }^{13}$, Roland Wiest ${ }^{13}$, Pascal Martin ${ }^{14}$, Raviteja Kotikalapudi ${ }^{14}$, Terence J. O'Brien ${ }^{15,16}$, Benjamin Sinclair ${ }^{15,16}$, Lucy Vivash ${ }^{15,16}$, Patricia M.

Desmond $^{16}$, Elaine Lui ${ }^{16}$, Anna Elisabetta Vaudano ${ }^{17,18}$, Stefano Meletti ${ }^{17,18}$, Manuela Tondelli ${ }^{18,19}$, Saud Alhusaini ${ }^{20,21}$, Colin P. Doherty ${ }^{22,23}$, Gianpiero L. Cavalleri ${ }^{20,23}$, Norman Delanty ${ }^{20,23}$, Reetta Kälviäinen ${ }^{24,25}$, Graeme D. Jackson ${ }^{26}$, Magdalena Kowalczyk ${ }^{26}$, Mario Mascalchi ${ }^{27}$, Mira Semmelroch ${ }^{26}$, Rhys H. Thomas ${ }^{28}$, Hamid Soltanian-Zadeh ${ }^{29,30}$, Esmaeil Davoodi-Bojd ${ }^{31}$, Junsong Zhang ${ }^{32}$, Barbara A. K. Kreilkamp ${ }^{9}$, Matteo Lenge ${ }^{33,34}$, Renzo Guerrini ${ }^{33}$, Khalid Hamandi ${ }^{35,36}$, Sonya Foley ${ }^{36}$, Theodor Rüber $37,38,39$, Bernd Weber ${ }^{40}$, Chantal Depondt ${ }^{41}$, Julie Absil ${ }^{42}$, Sarah J. A. Carr $^{46}$, Eugenio Abela ${ }^{43}$, Mark P. Richardson ${ }^{43}$, Orrin Devinsky ${ }^{44}$, Mariasavina Severino ${ }^{45}$, Pasquale Striano ${ }^{45,46}$, Domenico Tortora ${ }^{46}$, Sean N. Hatton ${ }^{47}$, Sjoerd B. Vos ${ }^{48,49,50}$, Lorenzo Caciagli 48,51 , John S. Duncann ${ }^{48,49}$, Christopher D. Whelan ${ }^{20}$, Paul M. Thompson ${ }^{52}$, Sanjay M. Sisodiya ${ }^{48,49}$, Andrea Bernasconii ${ }^{53}$, Angelo Labate ${ }^{2,3}$, Carrie R. McDonald ${ }^{54}$, Neda Bernasconi ${ }^{52}$, Boris C. Bernhardt ${ }^{1,}$

Epilepsy is associated with genetic risk factors and cortico-subcortical network alterations, but associations between neurobiological mechanisms and macroscale connectomics remain unclear. This multisite ENIGMA-Epilepsy study examined whole-brain structural covariance networks in patients with epilepsy and related findings to postmortem co-expression patterns of epilepsy risk genes. Brain network analysis included 578 adults with temporal lobe epilepsy (TLE), 288 adults with idiopathic generalized epilepsy (IGE), and 1,328 healthy controls from 18 centres worldwide. Graph theoretical analysis of structural covariance networks revealed increased clustering and path length in orbitofrontal and temporal regions in TLE, suggesting a shift towards network regularization. Conversely, people with IGE showed decreased clustering and path length in fronto-temporo-parietal cortices, indicating a random network configuration. Syndrome-specific topological alterations reflected expression patterns of risk genes for hippocampal sclerosis in TLE and for generalized epilepsy in IGE. These imaging-genetic signatures could guide diagnosis, and ultimately, tailor therapeutic approaches to specific epilepsy syndromes.
\end{abstract}

\footnotetext{
${ }^{1}$ Multimodal Imaging and Connectome Analysis Laboratory, McConnell Brain Imaging Centre, Montreal Neurological Institute and Hospital, McGill University, Montreal, QC, Canada; ${ }^{2}$ Neuroscience Research Center, University Magna Græcia, Catanzaro, CZ, Italy; ${ }^{3}$ Institute of Neurology, University Magna Græcia, Catanzaro, CZ, Italy; ${ }^{4}$ nstitute of Neurobiology, Universidad Nacional Autónoma de México, Querétaro, México; ${ }^{5}$ Institute of Systems, Molecular and Integrative Biology, University of Liverpool, Liverpool, United Kingdom; ${ }^{6}$ Walton Centre NHS Foundation Trust, Liverpool, United Kingdom; ${ }^{7}$ Department of Neurology, University of Campinas-UNICAMP, Campinas, São Paulo, Brazil; ${ }^{8}$ Department of Neurology, Medical University of South Carolina, Charleston, SC, USA; ${ }^{9}$ Department of Neurology, University of Medicine Göttingen, Göttingen, Germany; ${ }^{10}$ Institute of Diagnostic Radiology and Neuroradiology, Functional Imaging Unit, University Medicine Greifswald, Greifswald, Germany; ${ }^{11}$ Department of Neurology, University Medicine Greifswald, Greifswald, Germany; ${ }^{12}$ Institute of Diagnostic Radiology and Neuroradiology, University Medicine Greifswald, Greifswald, Germany; ${ }^{13}$ Support Center for Advanced Neuroimaging (SCAN), University Institute of Diagnostic and Interventional Neuroradiology, University Hospital Bern, Bern, Switzerland; ${ }^{14}$ Department of Neurology and Epileptology, Hertie Institute for Clinical Brain Research, University of Tübingen, Tübingen, Germany; ${ }^{15}$ Department of Neuroscience, Central Clinical School, Alfred Hospital, Monash University, Melbourne, Victoria, Australia; ${ }^{16}$ Departments of Medicine and Radiology, The Royal Melbourne Hospital, The University of Melbourne, Parkville, Victoria, Australia; ${ }^{17}$ Neurology Unit, OCB Hospital, Azienda Ospedaliera-Universitaria, Modena, Italy; ${ }^{18}$ Department of Biomedical, Metabolic and Neural Science, University of Modena and Reggio Emilia, Modena, Italy; ${ }^{19}$ Primary Care Department, Azienda Sanitaria Locale di Modena, Modena, Italy; ${ }^{20}$ Department of Molecular and Cellular Therapeutics, The Royal College of Surgeons in Ireland, Dublin, Ireland; ${ }^{21}$ Department of Neurology, Yale University School of Medicine, New Haven, CT, USA; ${ }^{22}$ Department of Neurology, St James' Hospital, Dublin, Ireland; ${ }^{23}$ FutureNeuro SFI Research Centre, Dublin, Ireland; ${ }^{24}$ Epilepsy Center, Neuro Center, Kuopio University Hospital, Member of the European Reference Network for Rare and Complex Epilepsies EpiCARE, Kuopio, Finland; ${ }^{25}$ Faculty of Health Sciences, School of Medicine, Institute of Clinical Medicine, University of Eastern Finland, Kuopio, Finland; ${ }^{26}$ Florey Institute of Neuroscience and Mental Health, University of Melbourne, Melbourne VIC 3010, Australia; ${ }^{27}$ Neuroradiology Research Program, Meyer Children Hospital of Florence, University of Florence, Florence, Italy; ${ }^{28}$ Transitional and Clinical Research Institute, Newcastle University, Newcastle upon Tyne, UK; ${ }^{29}$ Contol and Intelligent Processing Center of Excellence (CIPCE), School of Electrical and Computer Engineering, University of Tehran, Tehran, Iran; ${ }^{30}$ Departments of Research Administration and Radiology, Henry Ford Health System, Detroit, MI, USA; ${ }^{31}$ Department of Neurology, Henry Ford Health System, Detroit, MI, USA; ${ }^{32}$ Cognitive Science Department, Xiamen University, Xiamen, China; ${ }^{33}$ Child Neurology Unit and Laboratories, Neuroscience Department, Children's Hospital A. Meyer-University of Florence, Italy;

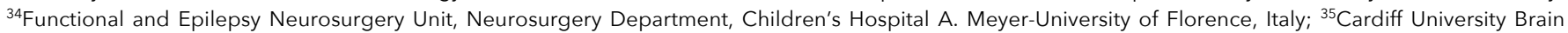
Research Imaging Centre (CUBRIC), College of Biomedical Sciences, Cardiff University, Cardiff, UK; ${ }^{36}$ The Welsh Epilepsy Unit, Department of Neurology, University Hospital of Whales, Cardiff, UK; ${ }^{37}$ Department of Epileptology, University of Bonn Medical Center, Bonn, Germany; ${ }^{38}$ Epilepsy Center Frankfurt RhineMain, Department of Neurology, Goethe-University Frankfurt, Frankfurt am Main, Germany; ${ }^{39}$ Center for Personalized Translational Epilepsy Research (CePTER), Goethe-University Frankfurt, Frankfurt am Main, Germany; ${ }^{40}$ Institute of Experimental Epileptology and Cognition Research, University Hospital Bonn, Bonn, Germany; ${ }^{41}$ Department of Neurology, Hôpital Erasme, Université Libre de Bruxelles, Brussels, Belgium; ${ }^{42}$ Department of Radiology, Hôpital Erasme, Université Libre de Bruxelles, Brussels, Belgium; ${ }^{43}$ Division of Neuroscience, Institute of Psychiatry, Psychology and Neuroscience, King's College London, UK; ${ }^{44}$ Department of Neurology, NYU Grossman School of Medicine, New York, NY, United States; ${ }^{45}$ IRCCS Istituto Giannina Gaslini, Genova, Italy; ${ }^{46}$ Department of Neurosciences, Rehabilitation, Ophthalmology, Genetics, Maternal and Child Health, University of Genova, Genova, Italy; ${ }^{47}$ Department of Neurosciences, Center for Multimodal Imaging and Genetics, University of California San Diego, La Jolla, CA, United States; ${ }^{48}$ Department of Clinical and Experimental Epilepsy, UCL Institute of Neurology, London, UK; ${ }^{49}$ Chalfont Centre for Epilepsy, Bucks, UK; ${ }^{50}$ Centre for Medical Image Computing, University College London, London, UK; ${ }^{51} \mathrm{MRI}$ Unit, Epilepsy Society, Chalfont St Peter, Bucks, UK; ${ }^{52}$ Imaging Genetics Center, Mark \& Mary Stevens Institute for Neuroimaging and Informatics, Keck School of Medicine, University of Southern California, Los Angeles, CA, United States; ${ }^{53}$ Neuroimaging of Epilepsy Laboratory, McConnell Brain Imaging Centre, Montreal Neurological Institute and Hospital, McGill University, Montreal, QC, Canada; ${ }^{54}$ Department of Psychiatry, Center for Multimodal Imaging and Genetics, University of California San Diego, La Jolla, CA, United States. e-mail: saratheriver@gmail.com; boris.bernhardt@mcgill.ca
} 


\section{INTRODUCTION}

$\mathrm{E}$ pilepsy is characterized by recurrent seizures and affects over 50 million people worldwide ${ }^{1}$. Cumulating evidence in epilepsy research has underscored the importance of interconnected brain networks in understanding the causes and consequences of the disease ${ }^{2,3}$. In the common epilepsies, particularly temporal lobe epilepsy (TLE) and idiopathic generalized epilepsy (IGE), histopathological and neuroimaging studies have demonstrated structural and functional compromise across widespread brain networks ${ }^{3,4,5,6,7}$. Magnetic resonance imaging (MRI) analysis of brain morphology, including cortical thickness measurements and grey matter volumetry, provide in vivo evidence of structural alterations across multiple cortical and subcortical regions in both TLE ${ }^{8,9}$ and IGE ${ }^{10,11}$. Beyond small cohort studies, robust patterns of atrophy across widespread brain networks were identified in the common epilepsies through the ENIGMA-Epilepsy (Enhancing NeuroImaging Genetics through MetaAnalysis) consortium, with data aggregated from multiple international sites ${ }^{12}$.

Covariation of morphological MRI markers, termed "structural covariance analysis," can extend earlier results on regional mapping of healthy and diseaserelated structural brain organization by identifying interregional network mechanisms. In healthy individuals, this approach has detected manifestations of functional crosstalk and structural connectivity between distributed regions ${ }^{13,14,15}$, and can tap into networks governed by shared genetic and maturational processes 16, 17, 18, 19, 20 . Prior work applying graph theoretical analyses to structural covariance networks has also characterized normative network topology ${ }^{21}$, revealing the presence of a "small world" organization. This architecture, which incorporates high clustering within segregated communities together with short paths between them, may provide a balance between network specialization and integration ${ }^{22}$. In TLE and IGE, structural covariance studies show syndrome-specific deviations from such a topological arrangement. In TLE, increased path length and clustering has been observed across both cortical systems ${ }^{23,24}$ and in limbic/paralimbic ${ }^{25}$ networks. In contrast, diverging topological alterations have been reported in IGE, echoing either global increases in clustering ${ }^{26,27}$ or path length ${ }^{28}$, global decreases in path length ${ }^{27}$, or no changes in network measures ${ }^{29}$. Analysis of structural brain metrics, gathered by ENIGMAEpilepsy, provides an opportunity to consolidate network alterations in the common epilepsies in a generalizable manner.
Interactions across multiple spatial scales, ranging from genetic factors to macroscale cortical morphology and structural networks, shape cortical and subcortical organization in both health and disease ${ }^{30}$. When combined, these naturally intertwined dimensions offer new insights into the pathophysiology of system-level disorders such as epilepsy ${ }^{31}$. Neuroimaging studies of large-scale networks can profit from studies on the landscape of genetic risk factors in common epilepsies 32, 33. Recently, the open release of postmortem human transcriptomics datasets, such as the Allen Human Brain Atlas (AHBA), has yielded opportunities to explore how gene co-expression patterns in the brain reflect macroscale neuroimaging findings 34,35 . Integrating imaging and genetics can shed light on the micro-tomacroscale mechanisms that underly the pathophysiology of the common epilepsies. In parallel, this combination can also be used to understand the ways in which genes may drive, to some extent, network alterations in epilepsy. How, and whether, structural covariance network properties converge with spatial expression patterns of risk genes for epilepsies, however, remains an unanswered question.

In this ENIGMA-Epilepsy study, we aimed to identify robust structural network disruptions in individuals with TLE and IGE relative to healthy controls, aggregating inter-regional cortical thickness and subcortical volume correlations across 18 international sites. Graph theoretical analysis assessed global and regional topological disruptions in both epilepsy syndromes. Moreover, we leveraged gene expression information from the AHBA to relate macroscale network findings to spatial expression patterns of genetic risk factors in these two major forms of epilepsy. Spatial associations between topological changes and disease-specific gene expression maps were evaluated against null models with similar spatial autocorrelations ${ }^{34,36}$. Reproducibility of our findings was also assessed across sites, variable network construction approaches, and clinical variables (side of seizure onset, disease duration).

\section{RESULTS}

\section{Data samples}

We studied 866 adult epilepsy patients (377 males, mean age $\pm \mathrm{SD}=33.82 \pm 9.48$ years) and 1,328 healthy controls (588 males, mean age $\pm \mathrm{SD}=30.74 \pm 8.30$ years) from 18 centres in the international Epilepsy Working Group of ENIGMA ${ }^{37}$. Our analyses focused on two patient subcohorts with site-matched healthy controls: TLE with neuroradiological evidence of hippocampal sclerosis 


\begin{tabular}{cccccc}
$\begin{array}{c}\text { Case-control } \\
\text { subcohorts }\end{array}$ & $\begin{array}{c}\text { Age } \\
(\text { mean } \pm \text { SD })\end{array}$ & $\begin{array}{c}\text { Age at onset } \\
(\text { mean } \pm \text { SD })\end{array}$ & $\begin{array}{c}\text { Sex } \\
(\text { male/female })\end{array}$ & $\begin{array}{c}\text { Side of focus } \\
(\mathrm{L} / \mathrm{R})\end{array}$ & $\begin{array}{c}\text { Duration } \\
\text { of illness } \\
(\text { mean } \pm \text { SD })\end{array}$ \\
\hline $\begin{array}{c}\text { TLE } \\
(n=578)\end{array}$ & $35.89 \pm 9.15$ & $15.09 \pm 11.23^{\mathrm{a}}$ & $267 / 311$ & $321 / 257$ & $21.12 \pm 13.02^{\mathrm{a}}$ \\
$\begin{array}{c}\mathrm{HC} \\
(n=1,083)\end{array}$ & $31.72 \pm 8.54$ & - & $490 / 593$ & - & - \\
\hline $\begin{array}{c}\mathrm{IGE} \\
(n=288)\end{array}$ & $29.65 \pm 8.75$ & $14.73 \pm 8.55^{\mathrm{a}}$ & $110 / 178$ & - & $14.46 \pm 10.86^{\mathrm{a}}$ \\
$\begin{array}{c}\mathrm{HC} \\
(n=911)\end{array}$ & $29.95 \pm 8.18$ & - & $385 / 526$ & - & - \\
\hline
\end{tabular}

TABLE 1. ENIGMA Epilepsy Working Group demographics. Demographic breakdown of patient-specific subcohorts with site-matched controls, including age (in years), age at onset of epilepsy (in years), sex, side of seizure focus (TLE patients only), and mean duration of illness (in years). Healthy controls from sites that did not have TLE (or IGE) patients

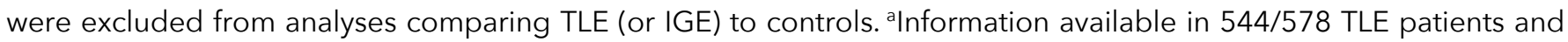
248/288 IGE patients.

$\left(n_{\mathrm{HC} / \mathrm{TLE}}=1,083 / 578,257\right.$ right-sided focus $)$ and IGE $\left(n_{\mathrm{HC} / \mathrm{IGE}}=911 / 288\right)$. Subject inclusion criteria and casecontrol subcohorts are detailed in the MATERIALS AND METHODS and TABLE 1. Site-specific demographic and clinical information are provided in TABLE S1. All participants were aged between 18-50 years.

\section{Global network characteristics}

Cortical thickness (measured across 68 gray matter brain regions ${ }^{38}$ ) and volumetric data (measured across 12 subcortical gray matter regions and bilateral hippocampi) were obtained from all patients and controls. Cortical and subcortical data were harmonized across scanners and sites using CovBat ${ }^{39}$, and statistically corrected for age, sex, mean cortical thickness, and intracranial volume ${ }^{39}$. Group- and sitespecific structural covariance networks were then computed from morphometric (cortical thickness/subcortical volume) correlations (FIG. 1A). To characterize the topology of structural covariance networks, we computed two fundamental and widely used graph-theoretical parameters ${ }^{40}$ : (i) mean clustering coefficient, to quantify local network efficiency, and (ii) mean path length, to index global efficiency (FIG. 1B).

Comparing patients with TLE to controls, our multisite analysis revealed modest increases in overall clustering coefficient $\left(p_{\text {uncorr }}<0.05\right.$ at network densities $(K)=0.08-0.11$; see METHODS) and decreases in mean path length over multiple density thresholds $\left(p_{\mathrm{FDR}}<0.1\right.$ at $K=0.05-0.15,17-18,22-23$, and $0.30-0.50$ ) in patients.
In contrast, IGE patients showed, on average, similar overall clustering coefficient relative to controls, but marginal decreases in overall path length $\left(p_{\text {uncorr }}<0.05\right.$ at $K=0.31-0.50$; FIG. 1C).

\section{Regional network characteristics}

We also quantified clustering coefficient and path length changes at the nodal level. Multivariate comparisons, combining clustering coefficient and path length in TLE relative to controls, revealed trends for topological alterations in bilateral parahippocampus, paracentral lobule, lateral occipital cortex, putamen, and caudate, ipsilateral angular gyrus and orbitofrontal cortex, as well as contralateral insula, middle, and inferior temporal gyri (all $p_{\mathrm{FDR}}<0.06$ ). Although regional Cohen's $d$ effect sizes estimated across sites revealed an overall increase in small-worldness in TLE (Cohen's $d$ mean \pm SD: clustering $=0.26 \pm 0.16$, path length $=0.19 \pm 0.13$ ), there were variations in different subnetworks. Notably, paralimbic and limbic regions such as the bilateral orbitofrontal, temporal, and angular cortices as well as ipsilateral amygdala showed an increase in clustering coefficient and path length (Cohen's $d$ mean \pm SD: clustering $=0.20 \pm 0.13$, path length $=0.18 \pm 0.11$ ), suggestive of a more regularized, "lattice-like," subnetwork arrangement (FIG. 2A).

When compared to controls, individuals with IGE showed widespread multivariate topological alterations in left inferior frontal gyrus pars opercularis, superior temporal sulcus, and nucleus accumbens, and right 
bioRxiv preprint doi: https://doi.org/10.1101/2021.10.18.464713; this version posted October 19, 2021. The copyright holder for this preprint (which was not certified by peer review) is the author/funder, who has granted bioRxiv a license to display the preprint in perpetuity. It is made available under aCC-BY-NC-ND 4.0 International license.

a | Construction of group- and site-specific structural covariance networks
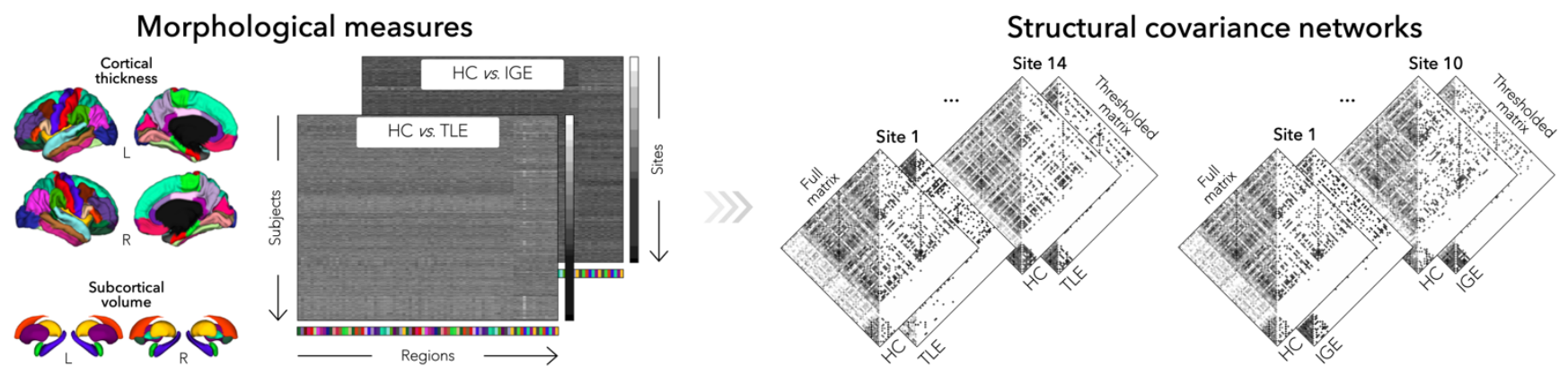

b | Graph theoretical parameters and topological properties
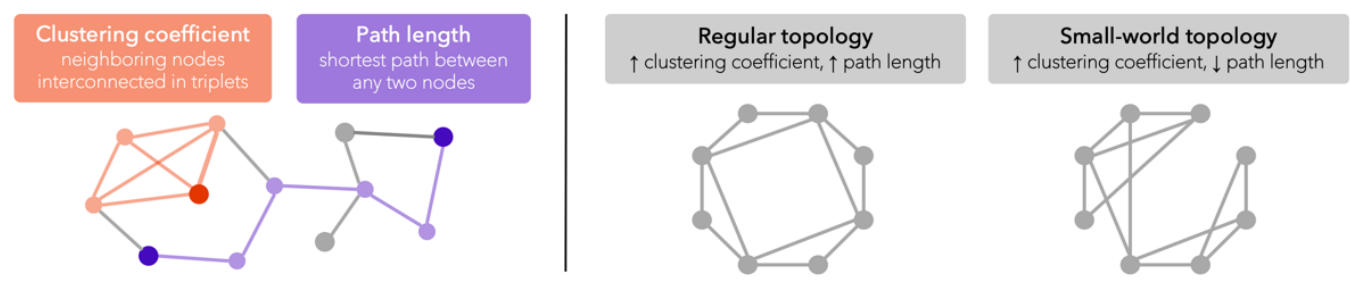

Randomized topology $\downarrow$ clustering coefficient, $\downarrow$ path length

c Global network alterations in the common epilepsies
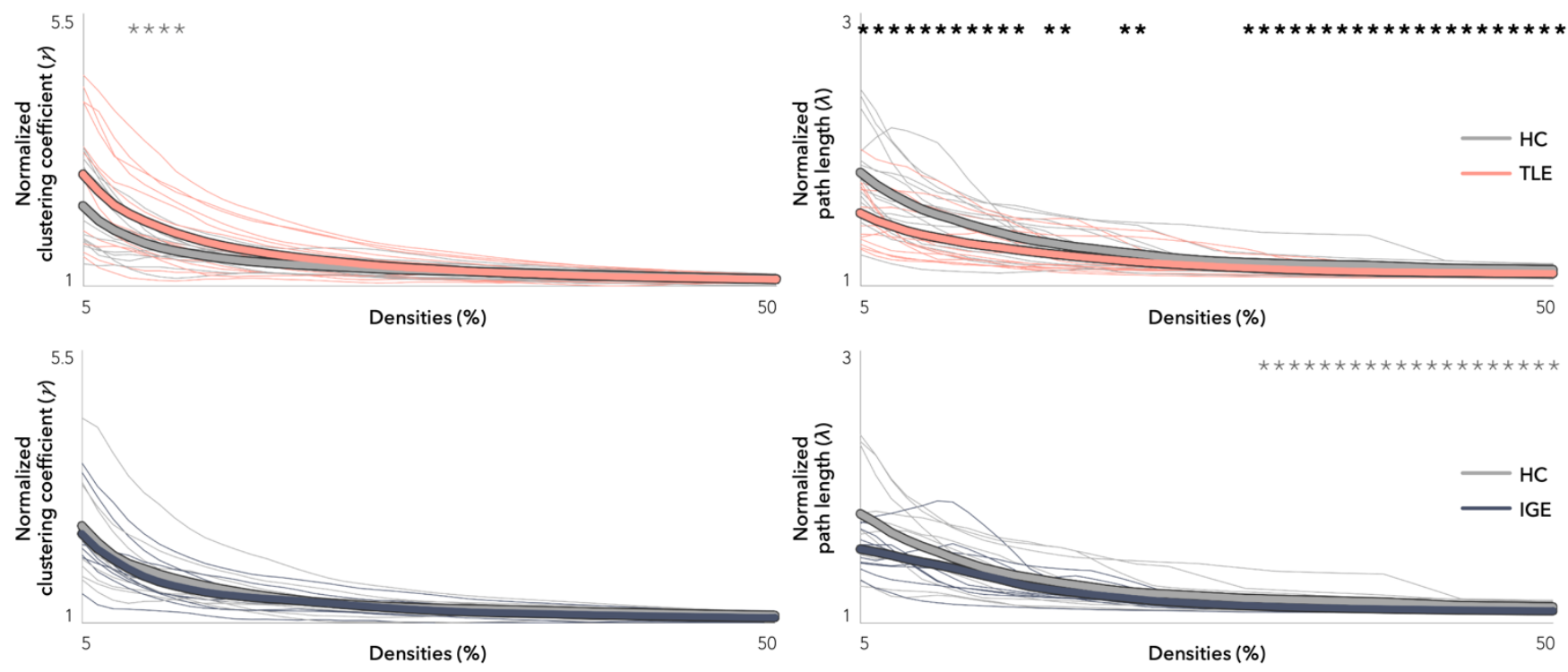

FIGURE 1. Structural covariance networks in the common epilepsies. (a) Schematic showing the construction of groupand site-specific structural covariance networks from morphometric correlations. (b) Two graph theoretical parameters characterized network topology: clustering coefficient, which measures connection density among neighboring nodes (orange) and path length, which measures the number of shortest steps between any two given nodes (purple). The interplay between clustering coefficient and path length can describe three distinct topological organizations: regular networks with high clustering and path length (left), small-world networks with high clustering and low path length (middle), and random networks with low clustering and path length (right). (c) Global differences in clustering coefficient (left) and path length (right) between TLE and HC (top) and between IGE and HC (bottom) are plotted as a function of network density. Increased small-worldness (i.e., increased clustering and decreased path length) was observed in individuals with TLE, whereas individuals with IGE showed decreases in clustering and path length, suggesting a more random configuration. Student's t-tests were performed at each density value; bold asterisks indicate $p_{F D R}<0.1$, semitransparent asterisks indicate $p_{\text {uncorr }}<0.05$. Thin lines represent data from individual sites. 
a | Topological network regularization in TLE

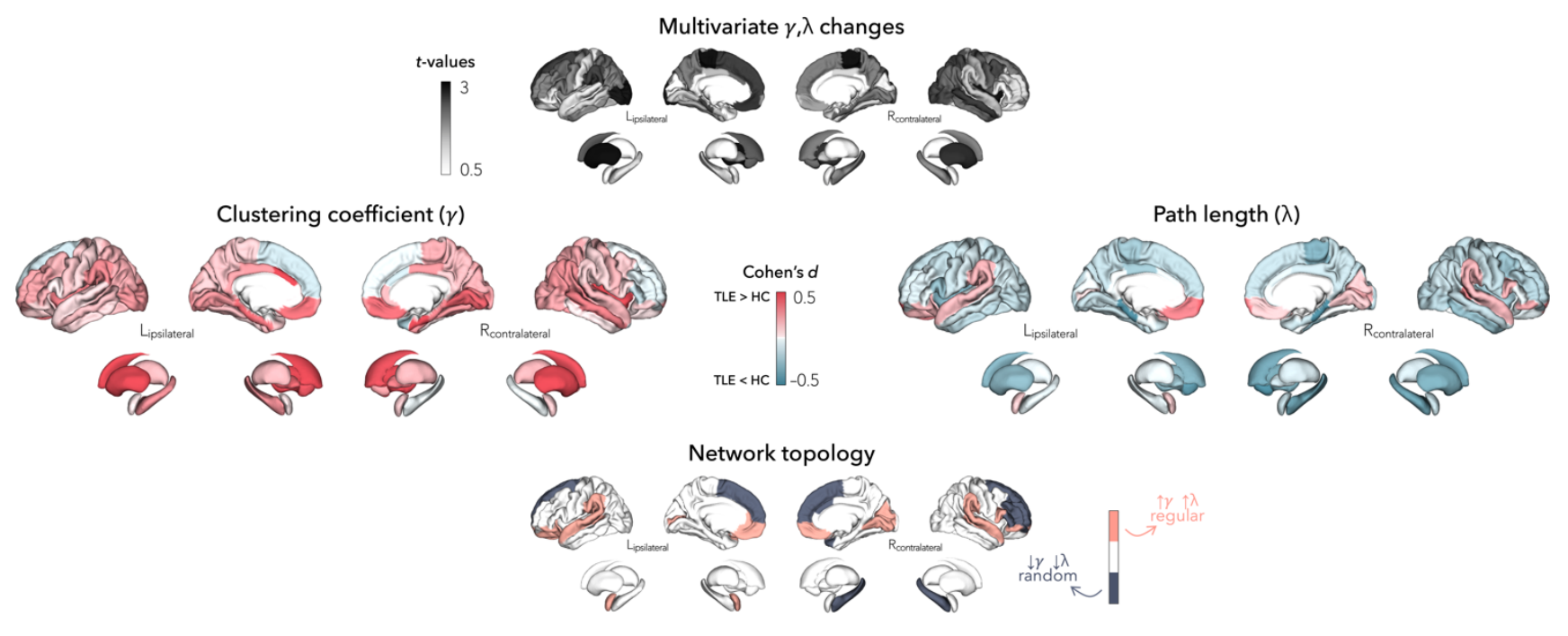

b | Topological network randomization in IGE

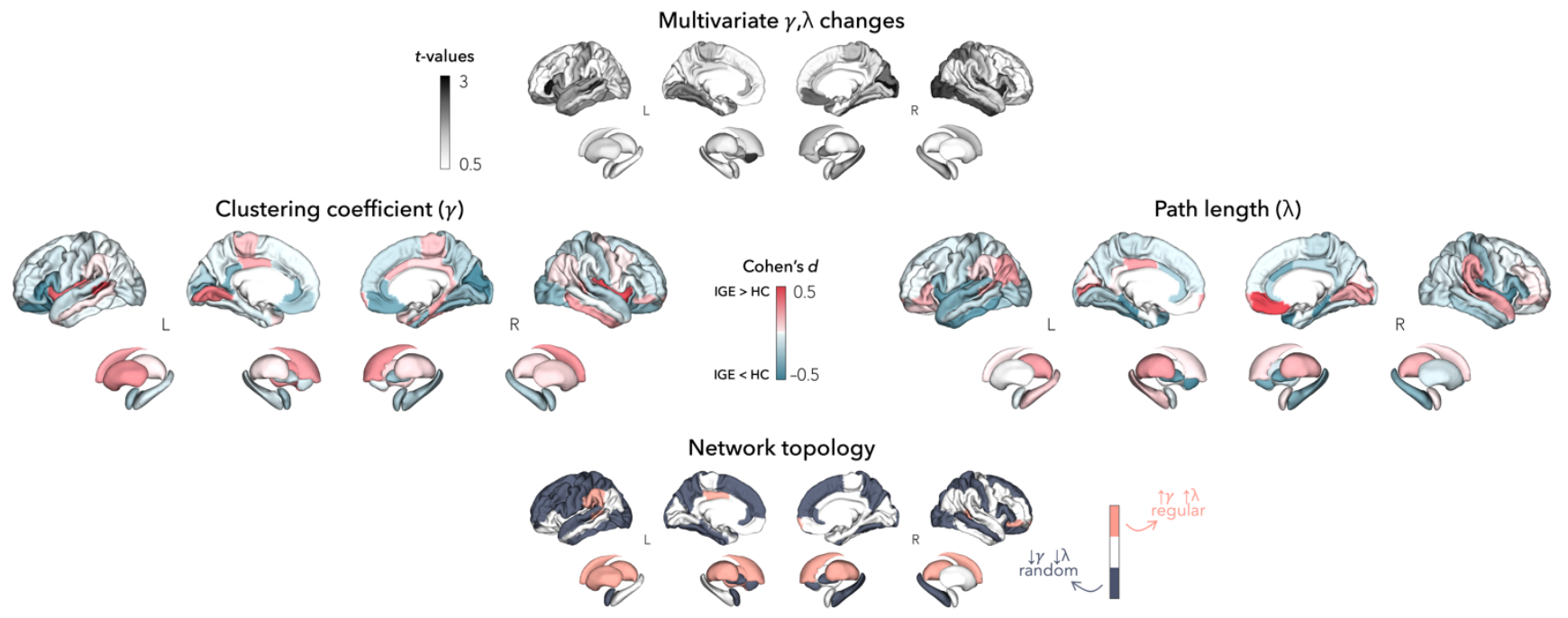

FIGURE 2. Nodal network alterations. (a) Graph theoretical analysis of structural covariance between individuals with TLE and controls revealed increased clustering and path length in bilateral orbitofrontal, temporal, and angular cortices, caudate, and putamen, as well as ipsilateral amygdala, revealing a regularized, "lattice-like," arrangement. (b) In IGE, widespread multivariate topological alterations were observed in bilateral fronto-temporo-parietal cortices, right nucleus accumbens, and left pallidum. Clustering and path length effect sizes in these regions suggest a randomized network configuration (decreased clustering and path length).

calcarine sulcus, insula, inferior temporal gyrus, and lateral occipital cortex (all $p_{\mathrm{FDR}}<0.013$ ). Effect sizes for each individual metric revealed decreased clustering coefficient and path length, with predominant changes in bilateral fronto-temporo-parietal cortices, nucleus accumbens, and pallidum (Cohen's $d$ mean \pm SD: clustering $=-0.15 \pm 0.11$, path length $=-0.22 \pm 0.17$ ), suggesting a more randomized network configuration (FIG. 2B). Increased small-worldness in IGE was also observed in fronto-parietal (bilateral paracentral lobule, right precentral gyrus) and temporal (left middle temporal gyrus, right inferior temporal gyrus) regions, but appeared to be primarily driven by conspicuously shorter path length (Cohen's $d$ mean $\pm \mathrm{SD}$ : clustering $=0.16 \pm 0.14$, path length $=-0.27 \pm 0.19$ ).

\section{Transcriptomic associations}

Having established multivariate topological abnormalities in TLE and IGE, we evaluated whether these network-level findings were associated with the 
a | Transcriptomic associations to epilepsy-related genes
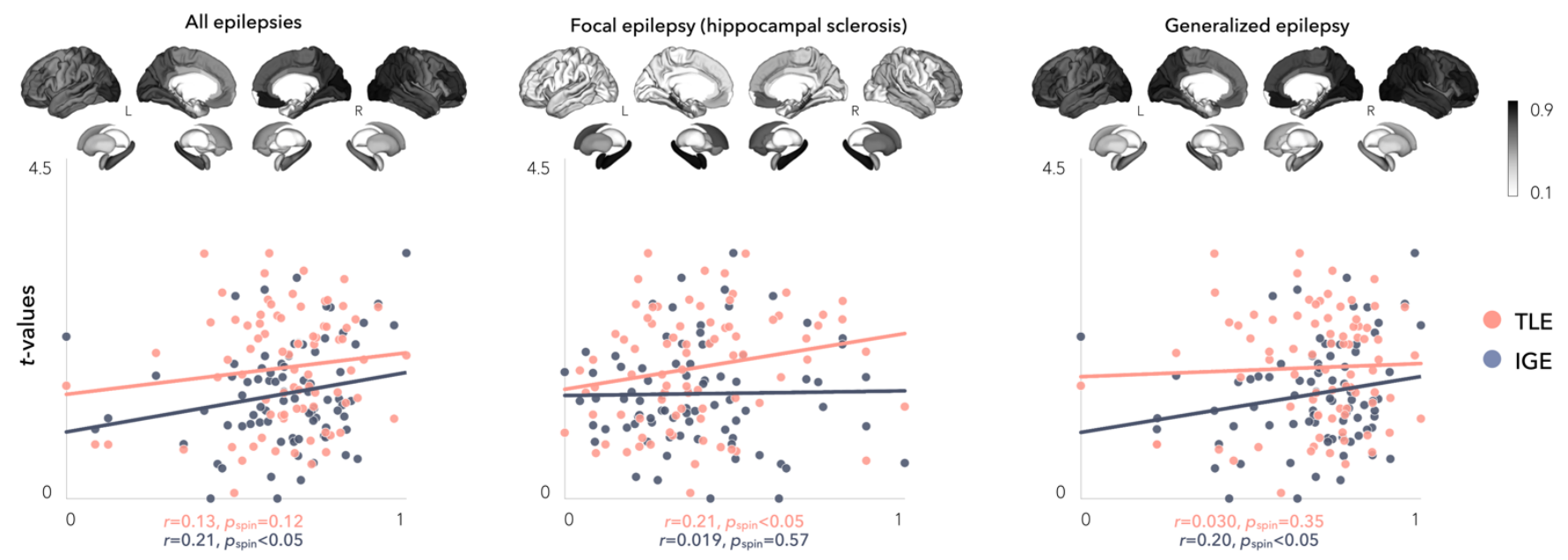

Gene co-expression levels

b | Transcriptomic associations to other disease-related risk genes
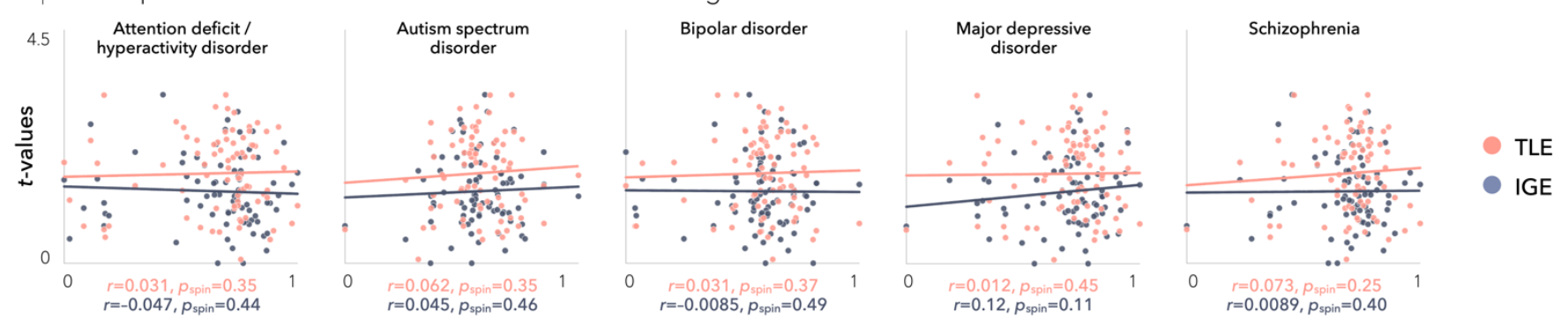

Gene co-expression levels

FIGURE 3. Relations between epilepsy gene co-expression and network topology. (a) Epilepsy-related gene coexpression levels are mapped to cortical and subcortical surface templates. Spatial associations between microarray data and multivariate topological changes were strongest for expression levels of hippocampal sclerosis genes in TLE $\left(r=0.21, p_{\text {spin }}<0.05\right)$ and generalized epilepsy genes in IGE $\left(r=0.20, p_{\text {spin }}<0.05\right)$. (b) Network associations did not correlate with other disease-related transcriptomic maps, suggesting specificity of these associations to epilepsyrelated molecular risk factors.

spatial expression patterns of previously established genetic risk factors. To this end, we assessed spatial correlations between epilepsy-related gene expression maps (genes listed in a recently published GWAS ${ }^{32}$ and co-expression levels obtained from the Allen Human Brain Atlas ${ }^{34}$; see MATERIALS AND Methods and TABLE S2) and multivariate topological profiles. To assess specificity, we also cross-referenced our network findings with five additional transcriptomic maps from common neuropsychiatric conditions, including: attention deficit/hyperactivity disorder ${ }^{41}$, autism spectrum disorder ${ }^{42}$, bipolar disorder ${ }^{43}$, major depressive disorder ${ }^{44}$, and schizophrenia ${ }^{45}$. Significance of spatial correlations was established using spin permutation tests ${ }^{36}$ that control for spatial autocorrelations (see MATERIALS AND METHODS), from the ENIGMA Toolbox [https://github.com/MICAMNI/ENIGMA; ${ }^{46}$.

We found significant associations between the spatial patterns of multivariate topological alterations in TLE and epilepsy risk gene co-expression levels of hippocampal sclerosis $\left(r=0.21, p_{\text {spin }}<0.05\right)$. On the other hand, multivariate topological differences in IGE were preferentially related to the co-expression levels of generalized epilepsy $\left(r=0.20, p_{\text {spin }}<0.05\right.$; FIG. 3A). In both TLE and IGE, network-level findings did not correlate with other disease-related transcriptomic maps (range $r=-0.047-0.12$, all $p_{\text {spin }}>0.1$; FIG. 3B).

\section{Associations to clinical variables}

As seizure focus laterality may differentially affect structural covariance networks ${ }^{23}$, we repeated the above analyses in left and right TLE independently, comparing 
patient subgroups to both controls and to each other. Global increases in clustering and decreases in path length were observed in both left (clustering: $p_{\text {FDR }}<0.05$ at $K=0.05-0.26,28-29$; path length: $p_{\mathrm{FDR}}<0.05$ at $K=0.05,0.07-0.22,25-39$; FIG. S1A) and right (clustering: $p_{\mathrm{FDR}}<0.05$ at $K=0.05-0.25$; path length: $p_{\text {uncorr }}<0.05$ at $K=0.14-0.17$; FIG. S2A) TLE patients relative to controls. Similarly, dominant patterns of multivariate (clustering coefficient and path length) topological changes in bilateral lateral occipital cortex $\left(p_{\mathrm{FDR}}<0.01\right)$, parahippocampus $\left(p_{\mathrm{FDR}}<0.005\right)$, entorhinal cortex $\left(p_{\mathrm{FDR}}<0.01\right)$, and insula $\left(p_{\text {uncorr }}<0.05\right)$, ipsilateral precuneus $\left(p_{\mathrm{FDR}}<0.01\right)$, anterior cingulate cortex $\left(p_{\mathrm{FDR}}<0.01\right)$, and superior temporal gyrus $\left(p_{\mathrm{FDR}}<0.05\right)$, as well as contralateral middle temporal gyrus $\left(p_{\mathrm{FDR}}<0.05\right)$ were observed when comparing left (FIG. S1A) and right (FIG. S2A) TLE cohorts separately to controls. Left TLE additionally showed alterations in bilateral paracentral and sensorimotor cortices $\left(p_{\mathrm{FDR}}<0.01\right)$, while right TLE additionally showed abnormalities in bilateral hippocampi $\left(p_{\mathrm{FDR}}<0.005\right)$. Direct comparison of left $v s$. right TLE revealed no significant global $\left(p_{\mathrm{FDR}}>0.27\right.$; FIG. S3A) nor regional $\left(p_{\mathrm{FDR}}>0.11\right.$; FIG. S3B $)$ differences between the two subcohorts. Effect sizes for clustering and path length indicated network regularization of the mesiotemporal and postcentral gyrus subnetwork in left TLE (FIG. S1A), but widespread cortical regularization in right TLE (FIG. S2A); these slight differences in regional topological configurations were further confirmed in left $v s$. right TLE comparisons (FIG. S3B). Differences in multivariate topological changes between left and right TLE marginally affected their associations to diseaserelated risk genes; in both groups, strongest spatial correlations were observed with co-expression levels of genes previously associated to hippocampal sclerosis (FIG. S1B, FIG. S2B).

We repeated the structural covariance analyses in patients grouped by duration of illness using a median split approach (TLE $=20$ years, $n_{\text {short-TLE }}=270, \quad n_{\text {long- }}$ TLE $=275 ;$ IGE $=15$ years, $\left.n_{\text {short-IGE }}=137, n_{\text {long-IGE }}=111\right)$. In TLE and IGE, both patient subgroups (short and long duration) showed similar patterns to the overall betweengroup differences when compared to controls (TLE: FIG. S4A; IGE: FIG. S5A). Nevertheless, in TLE, we observed a shift in network regularization from frontocentral and limbic regions (shorter duration) to frontotemporal and limbic regions (longer duration; FIG. S4B). Conversely, in IGE, we observed both network randomization (fronto-central regions) and regularization (fronto-parietal regions) in patients with short and long duration. Direct comparison of patients with short $v s$. long duration of TLE or IGE revealed no significant global (TLE: $p_{\mathrm{FDR}}>0.5$, FIG. S6A; IGE: $p_{\text {FDR }}>0.20$, FIG. S6B) nor regional (TLE: $p_{\text {FDR }}>0.072$, FIG. S6A) differences between pairs of subcohorts, with the exception of patients with shorter duration of IGE showing multivariate topological changes in bilateral fronto-limbic areas relative to those with longer duration $\left(p_{\mathrm{FDR}}<0.05\right.$, FIG. S6B $)$.

\section{Robustness of findings across different sites and analysis thresholds}

Despite some site-to-site variability, syndrome-specific global structural covariance differences were overall consistent across sites and similar to those obtained from the multisite aggregation for both TLE and IGE patients (FIG. 1C). As observed in the multisite findings, sitespecific increases in clustering coefficient and path length in TLE were most frequently observed in orbitofrontal, temporal, and angular cortices as well as amygdala (FIG. S7A). Similarly, in agreement with the multisite findings, site-specific decreases in clustering and path length in IGE were most consistent in frontoparietal cortices and hippocampus (FIG. S7B).

Our findings were not affected by varying the density of structural covariance networks: Across the range of possible thresholds, we observed high correlations among multivariate topological brain maps computed from thresholded structural covariance matrices in TLE $\left(95.35 \%\right.$ of correlations were below $\left.p_{\text {spin }}<0.1\right)$ and IGE ( $90.86 \%$ of correlations were below $p_{\text {spin }}<0.1$; FIG. S8A). Moreover, we observed comparable associations between topological abnormalities (computed across the range of thresholds) and gene co-expression levels, with highest stability in TLE observed for correlations of topological alterations and focal epilepsy with hippocampal sclerosis gene co-expression levels $\left(62.00 \%\right.$ of correlations were below $\left.p_{\text {spin }}<0.1\right)$. Conversely, stability in IGE was highest for correlations with gene co-expression levels of generalized epilepsy ( $40.00 \%$ of correlations were below $\left.p_{\text {spin }}<0.1\right)$, major depressive disorder $(42.00 \%$ of correlations were below $\left.p_{\text {spin }}<0.1\right)$, and schizophrenia $(52.00 \%$ of correlations were below $p_{\text {spin }}<0.1$; FIG. S8B).

\section{DISCUSSION}

This worldwide ENIGMA study is the largest investigation of structural covariance networks in the common epilepsies and bears robust evidence for syndrome-specific topological disruptions. First, despite showing global increases in small-worldness in TLE as compared to controls, profound regional alterations in 
orbito-fronto-temporal regions indicated a shift towards a more regularized, "lattice-like," subnetwork configuration. In contrast, IGE presented with widespread decreases in clustering and path length in fronto-temporo-parietal cortices, indicating a more random topology. These syndrome-specific networklevel findings were spatially related to the expression pattern of genetic risk factors associated with hippocampal sclerosis and generalized epilepsy in recent genome wide association studies (GWAS) ${ }^{32}$. Findings were highly consistent across sites and methodologies, corroborating robustness and generalizability. Taken together, our study identifies imaging-genetic signatures in the common epilepsies, which ultimately, may facilitate early diagnosis and lead to the development of new and improved treatment strategies.

We performed graph theoretical analysis on MRIbased cortical thickness and subcortical volume correlations in adults with TLE, adults with IGE, and healthy controls ${ }^{19,47}$. Our covariance analysis extends prior research on atrophy mapping by tapping into the topology of inter-regional structural brain networks and describing the network organization underlying wholebrain pathological interactions in the common epilepsies. Using a multisite approach, we showed that patients with TLE preserved an overall small-world configuration with increased clustering and decreased path length over a wide sparsity range. Upon examination of uni- and multivariate regional changes, however, we found key differences between distinct brain subnetworks. Topological alterations were most marked in a subnetwork comprising orbitofrontal, temporal, and angular cortices, pointing to increased local connectivity (i.e., a more regular configuration) in TLE than in healthy controls. This bilateral topological regularization was observed in both left and right TLE patients, albeit more constrained to fronto-temporal cortices in left TLE, a difference that may be attributable to asymmetrical structural damage or to higher connectivity of the dominant hemisphere ${ }^{48}$. Notably, when split into short $v s$. long duration groups, patients with longer duration of TLE exhibited topological regularization primarily in temporo-parietal cortices, areas that show progressive pathological changes unrelated to normal aging 3,49, 50,51. Although group-level alterations in the hippocampus were modest, with right TLE patients displaying slightly more severe abnormalities than left TLE, intrinsic hippocampal deafferentation may nevertheless contribute to extrahippocampal reconfigurations, affecting neighbouring regions including orbitofrontal and temporal cortices, as well as the amygdala ${ }^{52}$. Given the high density of connections from the hippocampus to the rest of the brain 53,54 , neuronal loss and deafferentation within limbic structures may cause local excess connectivity and decreased internetwork covariance in remote regions. Such a topological shift may be supported by findings in animal models ${ }^{55}$ as well as human diffusion MRI 52, 56, 57 and functional connectivity distance studies ${ }^{58}$, which have highlighted imbalances in short- vs. long-range connections in epilepsy-related pathology. More regularized networks are spatially compact, which may facilitate recurrent excitatory activity and high frequency oscillations, and may be attributable to a loss of temporo-limbic structural connections 55,59 . In line with prior EEG/intracranial EEG studies, network regularization has often been reported at seizure onset, a configuration that shifts toward a globally integrated process as the seizure spreads, eventually reaching a random configuration upon seizure termination ${ }^{60}$. Understanding such structural reorganization offers a comprehensive knowledge of the neural substrates and pathophysiological mechanisms of TLE.

In contrast with TLE, overall structural covariance network configurations in IGE showed a tendency away from aberrant local connectivity and towards a more random architecture. On the whole, increased randomness of the brain's structural network organization denotes reduced local efficiency but increased global efficiency ${ }^{21,}{ }^{61}$. These global topological findings were complemented by regionspecific mapping of graph-theoretical parameters, which also identified widespread regional alterations in IGE patients. Although the pattern was overall mixed across regions-a fortiori in smaller patient subgroups split according to disease duration-showing increases and decreases in both path length and clustering, a large subnetwork comprising frontal, temporal and parietal cortices showed concomitant reductions in path length as well as clustering. A decrease in clustering implies reductions in local specialization, but a decrease in path length (i.e., increased global network efficiency) may indicate an imbalance in the integration and segregation of structural covariance network organization. Such imbalance might explain, at least in part, the ability of seizures to rapidly spread, not just locally but in a diffuse manner within bilateral fronto-temporo-parietal cortices in IGE patients ${ }^{62}$. In rodent models of IGE, frontoparietal cortices have typically shown increased simultaneous neuronal activity during generalized seizures ${ }^{63,64}$. Moreover, extensive neuroimaging work in IGE patients that analyzed cortical morphology has shown widespread cortical structural network compromise ${ }^{6,65}$, with midline frontal and paracentral 
regions emerging as potential epicenters of morphological abnormalities in IGE ${ }^{3,66}$. Notably, IGE patients also presented with focal patches of network randomization, similar to TLE patients in paralimbic subnetworks. Affected regions included paralimbic cortices, but coupled with subcortical structures, notably the thalamus. Extensive evidence supports atypical thalamo-cortical interactions as being at the core of the pathophysiological network of IGE 7, 10, 11, 67, 68, with aberrant thalamo-cortical loops contributing to the generation of spike and slow wave discharges ${ }^{69}$. Alterations of thalamic morphology and metabolism, as well as of its functional and structural connectivity with widespread cortical networks, have also been reported in a convergent neuroimaging literature across several IGE syndromes ${ }^{10,70,71}$. In future work, it will be of interest to explore the consistency, or variability, of these topological imbalances in thalamic as well as cortical subnetworks are across different IGE subsyndromes. It is also important to understand how they vary with respect to other clinically relevant parameters including levels of drug-control. In that context, we recommend further increasing the spatial resolution, allowing for a fine-grained assessment of both cortical network architecture and thalamic subdivisions. This could be achieved, for example, by adopting recent approaches that reported structural, functional, and microcircuit anomalies in IGE compared to both TLE patients and healthy controls ${ }^{6,7}$.

Connectome topology has been extensively studied in healthy and diseased brains, however, research investigating associations between macroscale findings and the genetic architecture of epilepsy is still in infancy. A recent genome-wide mega-analysis performed in the common epilepsies identified 21 biological candidate genes across 16 risk loci, thus providing initial evidence for epilepsy-associated gene expression changes ${ }^{32}$. By integrating neuroimaging and transcriptional atlas data, here we tested the hypothesis that transcriptomic vulnerability would covary with structural network abnormalities in TLE and IGE. We showed that epilepsy-related variations in brain network topology spatially converged with gene expression profiles of risk genes for each syndrome. Specificity of these associations was further evidenced by the fact that topological alterations did not correlate with transcriptional signatures of several common psychiatric disorders. In the long term, these imaging-genetic associations may form the foundation for translation and clinical studies aiming to tailor therapeutic approaches to specific epilepsy syndromes. In parallel, our results may serve in the development of more effective treatments that can be targeted to the individual patients based on their genetic profile. Some limitations of the gene expression associations must be highlighted, including the fact that our understanding of the genetic architecture of the epilepsies is evolving, with the reported risk genes likely being expanded and refined as more genetic data in epileptic patients become available. Moreover, the gene expression information in the current work was derived from a small sample of six postmortem human donor brains, with predominant cortical and subcortical genetic microarray sampling performed in the left hemisphere. Even so, our findings suggest that genes previously associated with specific epilepsy syndromes were over-represented in regions that share similar topological alterations. In keeping with prior molecular studies in epilepsy 72,73,74,75, we speculate that differentially expressed epilepsy-related gene sets may contribute to a selective vulnerability of networks for structural reconfigurations in TLE and IGE. Replication of these distinctive imaging-genetic signatures in more comprehensive gene expression datasets may thus hold significant promise for stratification and effective treatment of epilepsy. Exploiting individualized gene expression profiles in the same cohort of patients, therefore, seems to be the logical next step to improve imaging-genetic associations and update our understanding of causes and consequences of epilepsy.

Several sensitivity analyses suggested that our findings were not affected by differences in scanners or sites or methodological choices. Site and scanner effects were mitigated for the most part using CovBat, a postacquisition statistical batch normalization process used to harmonize between-site and between-protocol effects in mean, variance, and covariance, while protecting biological covariates (e.g., disease status) ${ }^{39}$. Multivariate topological findings as well as associations between network-level findings and gene expression maps were consistently observed across different matrix thresholds. Despite some site-to-site variability in global and regional graph theoretical metrics, findings were overall similar across independent centers and reflected those from the multisite aggregation. As data sharing practices can at times be challenging, in part due to privacy and regulatory protection, ENIGMA represents a practical alternative for standardized data processing and anonymized derivative data $12,76,77,78,79,80$. This collaborative effort allowed us to identify a robust association of brain structural network changes with patterns of expression of genetic risk factors in the common epilepsies, while addressing robustness of effects across clinical subgroups, international sites, and methodological variations. The imaging-genetic 
associations identified herein could guide diagnosis of common epilepsies, and ultimately, contribute to the development of tailored, individualized, and syndromespecific therapeutic approaches.

\section{MATERIALS AND METHODS \\ ENIGMA participants}

Epilepsy specialists at each center diagnosed patients according to the seizure and syndrome classifications of the International League Against Epilepsy (ILAE). Inclusion of adults with TLE was based on the combination of electroclinical features and MRI findings typically associated with underlying hippocampal sclerosis. Inclusion of adults with IGE was based on the presence of tonic-clonic, absence, or myoclonic seizures with generalized spike-wave discharges on EEG. We excluded participants with a progressive or neurodegenerative disease (e.g., Rasmussen's encephalitis, progressive myoclonus epilepsy), malformations of cortical development, tumors, or prior neurosurgery. Local institutional review boards and ethics committees approved each included cohort study, and written informed consent was provided according to local requirements.

\section{Cortical thickness and subcortical volume data}

All participants underwent structural T1-weighted brain MRI scans at each of the 18 participating centers, with scanner descriptions and acquisition protocols detailed elsewhere ${ }^{37}$. Images were independently processed by each center using the standard ENIGMA workflow. In brief, models of cortical and subcortical surface morphology were generated with FreeSurfer 5.3.0 ${ }^{81}$. Based on the Desikan-Killiany anatomical atlas ${ }^{38}$, cortical thickness was measured across 68 grey matter brain regions and volumetric measures were obtained from 12 subcortical grey matter regions (bilateral amygdala, caudate, nucleus accumbens, pallidum, putamen, thalamus) as well as bilateral hippocampus. Missing cortical thickness and subcortical volume data were imputed with the mean value for that given region; participants with missing data in at least half of the cortical or subcortical brain measures were excluded.

Data were harmonized across scanners and sites using CovBat - a batch-effect correction tool that uses a Bayesian framework to improve the stability of the parameter estimates ${ }^{39}$. Cortical thickness and volumetric measures were corrected for age and sex. Residualized data were $z$-scored relative to site-matched pooled controls and sorted into measures that were ipsilateral/contralateral to the focus.

\section{Covariance networks}

Covariance networks were computed from cortical thickness and subcortical volume correlations. Interregional association matrices were first generated for each group (TLE, $\mathrm{HC}_{\mathrm{TLE}}$, IGE, $\mathrm{HC}_{\mathrm{IGE}}$ ) and each site with at least 10 participants per diagnostic group $\left(n_{\mathrm{TLE} / \mathrm{HC}}=15\right.$ sites, $n_{\mathrm{GE} / \mathrm{HC}}=10$ sites), resulting in a total of 50 covariance matrices $(R)$. In each matrix $R$, an individual entry $R i, j$ (with regions $i$ and $j$ ) contained the pairwise linear product-moment cross-correlation coefficient of structural morphometry across group- and site-specific subjects.

\section{Network thresholding}

Prior to analysis, negative correlations were set to zero and covariance network matrices were thresholded (density range of $K=0.05-0.50$, density interval of 0.01 ). This approach ensured that networks in all groups had an identical number of edges ${ }^{82}$ and that group differences were not primarily driven by low-level correlations ${ }^{83}$.

Among the network density levels of $K=0.05-0.50$, the network connectedness criterion $(\geq 75 \%$ of nodes remain connected to other nodes within the network in at least $90 \%$ of sites) was satisfied only in the narrower $K=0.08-0.50$ range. For the main regional topological analyses, structural networks were constructed at a density of $K=0.08$.

\section{Global network properties}

From the thresholded structural covariance networks, we computed two global metrics ${ }^{84}$ : (i) mean clustering coefficient, which quantifies the tendency for brain regions to be locally interconnected with neighboring regions, and (ii) mean path length, which quantifies the mean minimum number of edges (i.e., connection between two regions) that separate any two regions in the network. Each measure was normalized relative to corresponding measures from 1,000 randomly generated networks with similar degree and weight properties, and subsequently averaged across all cortical and subcortical regions, separately.

\section{Regional network properties}

Regional differences in topological parameters were assessed using an approach similar to the global network analysis; from the thresholded structural covariance networks, normalized clustering coefficient and normalized path length metrics were computed for every cortical and subcortical brain region. Individual nodal 
network parameters in patients were compared to controls across sites via Cohen's $d$ effect sizes. From effect size maps, topological profiles were generated to reflect either network regularization (areas of increased clustering coefficient and path length) or randomization (areas of decreased clustering coefficient and path length) ${ }^{85}$. Multivariate surface-based linear models were subsequently used to compare the aggregate of clustering coefficient and path length differences in patients relative to controls.

\section{Transcriptomic associations}

The Allen Institute for Brain Science released the AHBA - a brain-wide gene expression atlas comprising microarray expression data from over 20,000 genes sampled across 3,702 spatially distinct tissue samples collected from six human donor brains ${ }^{34}$. Microarray expression data were first generated using abagen ${ }^{86}$, a toolbox that provides reproducible workflows for processing and preparing gene co-expression data according to previously established recommendations ${ }^{87}$. Preprocessing steps included intensity-based filtering of microarray probes, selection of a representative probe for each gene across both hemispheres, matching of microarray samples to brain parcels from the DesikanKilliany atlas ${ }^{38}$, normalization, and aggregation within parcels and across donors. Genes whose similarity across donors fell below a threshold $(r<0.2)$ were removed, leaving a total of 12,668 genes for analysis.

Leveraging a recently published GWAS from the International League Against Epilepsy Consortium on Complex Epilepsies ${ }^{32}$, we extracted the most likely genes associated with significant genome-wide loci in the common epilepsies (i.e., all epilepsies, $n_{\text {genes }}=20$, as well as in two epilepsy syndromes (i.e., focal epilepsy with hippocampal sclerosis, $n_{\text {genes }}=3$ and generalized epilepsy, $n_{\text {genes }}=13$ ). We also queried additional lists of disease-related genes (obtained from other recently published GWAS), including gene sets for attention deficit/hyperactivity disorder $\left[n_{\text {genes }}=17^{41}\right]$, autism spectrum disorder $\left[n_{\text {genes }}=22^{42}\right]$, bipolar disorder $\left[n_{\text {genes }}=27^{43}\right]$, major depressive disorder $\left[n_{\text {genes }}=194^{44}\right]$, and schizophrenia $\left[n_{\text {genes }}=150^{45}\right]$. All gene sets were mapped to cortical and subcortical regions using the Allen Human Brain Atlas ${ }^{34}$ and projected to surface templates.

\section{Spatial permutation tests}

The intrinsic spatial smoothness in two given brain maps may inflate the significance of their spatial correlation, if the spatial dependencies in the data are not taken into account ${ }^{36}$. Statistical significance of spatial correlations (e.g., between multivariate topological patterns and transcriptomic maps) was assessed using spin permutation tests ${ }^{46}$. This framework generates null models of overlap between cortical maps by projecting the spatial coordinates of cortical and subcortical data onto the surface spheres (i.e., the parameter spaces), applying randomly sampled rotations (10,000 repetitions unless specified otherwise), and reassigning original values ${ }^{36}$. The empirical correlation coefficients are then compared against the null distribution determined by the ensemble of spatially permuted correlation coefficients.

\section{Associations to clinical variables}

As seizure focus lateralization may differentially impact topological organization of structural covariance networks ${ }^{24}$, we repeated the graph theoretical and transcriptomics analyses by comparing (i) left $\left(n_{\mathrm{LTLE}}=321\right)$ and right $\left(n_{\mathrm{RTLE}}=257\right)$ TLE independently to controls and (ii) directly comparing left $v$ s. right TLE.

To study the effects of duration of illness on structural covariance networks, we repeated the graph theoretical analyses by comparing $(i)$ patients with short duration of TLE or IGE (TLE duration $<20$ years, $n_{\text {short-TLE }}=270$; IGE duration $<15$ years, $\left.n_{\text {short-IGE }}=137\right)$ and patients with long duration of TLE or IGE (TLE duration $\geq 20$ years, $n_{\text {long- }}$ TLE $=275 ; \quad$ IGE duration $\geq 15$ years, $n_{\text {long-lGE }}=111$ ) independently to controls, (ii) directly comparing short vs. long TLE, and (iii) directly comparing short vs. long IGE. Median splits were used to group short $v s$. long duration patients.

\section{Reproducibility and sensitivity analyses}

Reproducibility across sites. To address reproducibility of our findings across different sites, we repeated our multivariate topological analysis independently in each site $\left(n_{\mathrm{TLE} / \mathrm{HC}}=14\right.$ sites, $n_{\mathrm{IGE} / \mathrm{HC}}=10$ sites $)$.

Stability across matrix thresholds. To verify that results were not biased by choosing a particular threshold, we repeated the network analyses and associations with disease-related gene co-expression levels across the range of matrix thresholds $(K=0.05-$ 0.50 with increments of 0.01 ). Specifically, Hotelling's $\mathrm{T}^{2}$ multivariate (clustering coefficient and path length) topological changes comparing patients to controls were computed from structural covariance networks thresholded at every density and pairwise spatial correlations between all pairs of multivariate brain maps were performed. Spatial correlations between densityspecific multivariate topological alterations and all disease-related transcriptomic maps were also assessed. Significance testing of these correlations was assessed via spin permutation tests with 1,000 repetitions. 


\section{ACKNOWLEDGEMENTS}

The authors would like to express their gratitude to the open science initiatives that made this work possible: $(i)$ the ENIGMA Consortium (core funding for ENIGMA was provided by the NIH Big Data to Knowledge (BD2K) program under consortium grant U54 EB020403 to P.M.T.) and (ii) The Allen Human Brain Atlas and the abagen toolbox (https://doi.org/10.5281/zenodo.4984124).

Sara Larivière was funded by the Fonds de la Recherche du Québec - Santé (FRQ-S), Canadian Institutes of Health Research (CIHR), and the Ann and Richard Sievers Neurosciene Award. Raul RodríguezCruces was funded by the FRQ-S. Jessica Royer was supported by a Canadian Open Neuroscience Platform (CONP) fellowship and CIHR. Lorenzo Caciagli acknowledges support from a Berkeley Fellowship jointly awarded by UCL and Gonville and Caius College, Cambridge, and by Brain Research UK (award 14181). The UNAM site was funded by UNAMDGAPA (IB201712, IG200117) and Conacyt (181508 and Programa de Laboratorios Nacionales). Mark Richardson was funded by UK Medical Research Council grant MR/K013998/1. Fernando Cendes and Clarissa Yasuda were supported by the São Paulo Research Foundation (FAPESP), Grant \# 2013/07559-3 (BRAINN - Brazilian Institute of Neuroscience and Neurotechnology). Stefano Meletti and Anna Elisabetta Vaudano were supported by the Ministry of Health (MOH), grant \# NET-2013-02355313. Paul Thompson was funded by R01 NS106957 and P41 EB015922. Carrie R. McDonald was supported by ENIGMA-R21 (NIH/NINDS R21NS107739) and R01 (NIH/NINDS R01 NS122827). Sanjay M. Sisodiya was supported by the Epilepsy Society, UK. Boris C. Bernhardt acknowledges research support from the National Science and Engineering Research Council of Canada (NSERC Discovery-1304413), the CIHR (FDN-154298, PJT-174995), SickKids Foundation (NI17-039), Azrieli Center for Autism Research (ACAR-TACC), BrainCanada, FRQ-S, and the Tier-2 Canada Research Chairs program.

\section{ETHICS DECLARATIONS}

Paul M. Thompson is funded in part by a research grant from Biogen, Inc. for work unrelated to the current manuscript.

\section{REFERENCES}

1. Feigin VL, et al. Global, regional, and national burden of neurological disorders, 1990-2016: a systematic analysis for the Global Burden of Disease Study 2016. The Lancet Neurology 18, 459-480 (2019).

2. Engel J, Jr., Thompson PM, Stern JM, Staba RJ, Bragin A, Mody I. Connectomics and epilepsy. Curr Opin Neurol 26, 186-194 (2013).

3. Lariviere $\mathrm{S}$, et al. Network-based atrophy modeling in the common epilepsies: A worldwide ENIGMA study. Sci Adv 6, (2020).

4. Bernhardt BC, et al. The spectrum of structural and functional imaging abnormalities in temporal lobe epilepsy. Ann Neurol 80, 142-153 (2016).

5. Concha L, Kim H, Bernasconi A, Bernhardt BC, Bernasconi N. Spatial patterns of water diffusion along white matter tracts in temporal lobe epilepsy. Neurology 79, 455-462 (2012).

6. Wang $\mathrm{Z}$, et al. Community-informed connectomics of the thalamocortical system in generalized epilepsy. Neurology 93, e1112-e1122 (2019).

7. Weng Y, et al. Macroscale and microcircuit dissociation of focal and generalized human epilepsies. Communications biology 3, 1-11 (2020).

8. Labate A, Cerasa A, Aguglia U, Mumoli L, Quattrone A, Gambardella A. Voxel-based morphometry of sporadic epileptic patients with mesiotemporal sclerosis. Epilepsia 51, 506-510 (2010).

9. McDonald CR, et al. Regional neocortical thinning in mesial temporal lobe epilepsy. Epilepsia 49, 794-803 (2008).

10. Bernhardt BC, Rozen DA, Worsley KJ, Evans AC, Bernasconi $\mathrm{N}$, Bernasconi A. Thalamo-cortical network pathology in idiopathic generalized epilepsy: insights from MRI-based morphometric correlation analysis. Neuroimage 46, 373-381 (2009).

11. O'Muircheartaigh J, et al. Abnormal thalamocortical structural and functional connectivity in juvenile myoclonic epilepsy. Brain 135, 3635-3644 (2012).

12. Sisodiya SM, et al. The ENIGMA-Epilepsy working group: Mapping disease from large data sets. Hum Brain Mapp, (2020).

13. Mechelli A, Friston KJ, Frackowiak RS, Price CJ. Structural covariance in the human cortex. The Journal of neuroscience : the official journal of the Society for Neuroscience 25, 83038310 (2005).

14. Gong G, He Y, Chen ZJ, Evans AC. Convergence and divergence of thickness correlations with diffusion connections across the human cerebral cortex. Neuroimage 59, 1239-1248 (2012).

15. Alexander-Bloch A, Raznahan A, Bullmore E, Giedd J. The Convergence of Maturational Change and Structural Covariance in Human Cortical Networks. The Journal of neuroscience : the official journal of the Society for Neuroscience 33, 2889-2899 (2013).

16. Zielinski BA, Gennatas ED, Zhou J, Seeley WW. Networklevel structural covariance in the developing brain. Proceedings of the National Academy of Sciences of the United States of America 107, 18191-18196 (2010).

17. Schmitt JE, et al. Variance decomposition of MRI-based covariance maps using genetically informative samples and structural equation modeling. Neuroimage 47, 56-64 (2009).

18. Khundrakpam BS, et al. Developmental Changes in Organization of Structural Brain Networks. Cereb Cortex, (2012). 

available under aCC-BY-NC-ND 4.0 International license.

19. Lerch JP, et al. Mapping anatomical correlations across cerebral cortex (MACACC) using cortical thickness from MRI. Neuroimage 31, 993-1003 (2006).

20. Raznahan A, et al. Patterns of coordinated anatomical change in human cortical development: a longitudinal neuroimaging study of maturational coupling. Neuron 72, 873-884 (2011).

21. Bullmore E, Sporns O. Complex brain networks: graph theoretical analysis of structural and functional systems. Nat Rev Neurosci 10, 186-198 (2009).

22. Sporns O, Chialvo DR, Kaiser M, Hilgetag CC. Organization, development and function of complex brain networks. Trends in cognitive sciences 8, 418-425 (2004).

23. Bernhardt BC, Chen Z, He Y, Evans AC, Bernasconi N. Graphtheoretical analysis reveals disrupted small-world organization of cortical thickness correlation networks in temporal lobe epilepsy. Cereb Cortex 21, 2147-2157 (2011).

24. Yasuda CL, et al. Aberrant topological patterns of brain structural network in temporal lobe epilepsy. Epilepsia 56, 1992-2002 (2015).

25. Bernhardt BC, Bernasconi N, Hong S, Dery S, Bernasconi A. Subregional mesiotemporal network topology is altered in temporal lobe epilepsy. Cereb Cortex 26, 3237-3248 (2016).

26. Liao W, et al. Relationship between large-scale functional and structural covariance networks in idiopathic generalized epilepsy. Brain connectivity 3, 240-254 (2013).

27. Bonilha L, et al. Neurodevelopmental alterations of large-scale structural networks in children with new-onset epilepsy. Human brain mapping 35, 3661-3672 (2014).

28. Lee HJ, Park KM. Structural and functional connectivity in newly diagnosed juvenile myoclonic epilepsy. Acta Neurologica Scandinavica 139, 469-475 (2019).

29. Sone D, et al. Reduced resilience of brain gray matter networks in idiopathic generalized epilepsy: A graph-theoretical analysis. PloS one 14, e0212494 (2019).

30. Betzel RF, Bassett DS. Multi-scale brain networks. Neuroimage 160, 73-83 (2017).

31. Lariviere S, et al. Multiscale Structure-Function Gradients in the Neonatal Connectome. Cereb Cortex, (2019).

32. Consortium TILAE. Genome-wide mega-analysis identifies 16 loci and highlights diverse biological mechanisms in the common epilepsies. Nature communications 9, (2018).

33. Leu C, et al. Pleiotropy of polygenic factors associated with focal and generalized epilepsy in the general population. PloS one 15, e0232292 (2020).

34. Hawrylycz MJ, et al. An anatomically comprehensive atlas of the adult human brain transcriptome. Nature 489, 391-399 (2012).

35. Fornito A, Arnatkevičiūtė A, Fulcher BD. Bridging the gap between connectome and transcriptome. Trends in Cognitive Sciences 23, 34-50 (2019).

36. Alexander-Bloch AF, et al. On testing for spatial correspondence between maps of human brain structure and function. Neuroimage 178, 540-551 (2018).

37. van Erp TGM, et al. Cortical Brain Abnormalities in 4474 Individuals With Schizophrenia and 5098 Control Subjects via the Enhancing Neuro Imaging Genetics Through Meta Analysis (ENIGMA) Consortium. Biol Psychiatry 84, 644-654 (2018).

38. Desikan RS, et al. An automated labeling system for subdividing the human cerebral cortex on MRI scans into gyral based regions of interest. Neuroimage 31, 968-980 (2006).

39. Chen AA, Beer JC, Tustison NJ, Cook PA, Shinohara RT, Shou H. Removal of scanner effects in covariance improves multivariate pattern analysis in neuroimaging data. bioRxiv, 858415 (2020).
40. Watts DJ, Strogatz SH. Collective dynamics of 'smallworld'networks. nature 393, 440-442 (1998).

41. Demontis D, et al. Discovery of the first genome-wide significant risk loci for attention deficit/hyperactivity disorder. Nature genetics 51, 63-75 (2019).

42. Grove J, et al. Identification of common genetic risk variants for autism spectrum disorder. Nature genetics 51, 431-444 (2019).

43. Stahl EA, et al. Genome-wide association study identifies 30 loci associated with bipolar disorder. Nature genetics 51, 793803 (2019).

44. Howard DM, et al. Genome-wide meta-analysis of depression identifies 102 independent variants and highlights the importance of the prefrontal brain regions. Nature neuroscience 22, 343-352 (2019).

45. Pardiñas AF, et al. Common schizophrenia alleles are enriched in mutation-intolerant genes and in regions under strong background selection. Nature genetics 50, 381-389 (2018).

46. Lariviere S, et al. The ENIGMA Toolbox: Cross-disorder integration and multiscale neural contextualization of multisite neuroimaging datasets. bioRxiv, (2020).

47. Alexander-Bloch A, Giedd JN, Bullmore E. Imaging structural co-variance between human brain regions. Nature Reviews Neuroscience 14, 322-336 (2013).

48. Bonilha L, et al. Asymmetrical extra-hippocampal grey matter loss related to hippocampal atrophy in patients with medial temporal lobe epilepsy. Journal of Neurology, Neurosurgery \& Psychiatry 78, 286-294 (2007).

49. Galovic M, et al. Progressive Cortical Thinning in Patients With Focal Epilepsy. JAMA Neurol, (2019).

50. Bernhardt BC, Worsley KJ, Kim H, Evans AC, Bernasconi A, Bernasconi N. Longitudinal and cross-sectional analysis of atrophy in pharmacoresistant temporal lobe epilepsy. Neurology 72, 1747-1754 (2009).

51. Caciagli L, Bernasconi A, Wiebe S, Koepp MJ, Bernasconi N, Bernhardt BC. A meta-analysis on progressive atrophy in intractable temporal lobe epilepsy: Time is brain? Neurology 89, 506-516 (2017).

52. Bonilha L, et al. Extrahippocampal gray matter loss and hippocampal deafferentation in patients with temporal lobe epilepsy. Epilepsia 51, 519-528 (2010).

53. de Wael RV, et al. Anatomical and microstructural determinants of hippocampal subfield functional connectome embedding. Proceedings of the National Academy of Sciences 115, 10154-10159 (2018).

54. Van Strien N, Cappaert N, Witter M. The anatomy of memory: an interactive overview of the parahippocampal-hippocampal network. Nature reviews neuroscience 10, 272-282 (2009).

55. Knopp A, Kivi A, Wozny C, Heinemann U, Behr J. Cellular and network properties of the subiculum in the pilocarpine model of temporal lobe epilepsy. Journal of Comparative Neurology 483, 476-488 (2005).

56. Concha L, Beaulieu C, Gross DW. Bilateral limbic diffusion abnormalities in unilateral temporal lobe epilepsy. Annals of neurology 57, 188-196 (2005).

57. Concha L, Livy DJ, Beaulieu C, Wheatley BM, Gross DW. In vivo diffusion tensor imaging and histopathology of the fimbria-fornix in temporal lobe epilepsy. Journal of Neuroscience 30, 996-1002 (2010).

58. Lariviere $\mathrm{S}$, et al. Functional connectome contractions in temporal lobe epilepsy: Microstructural underpinnings and predictors of surgical outcome. Epilepsia, (2020).

59. Sharma AK, Reams RY, Jordan WH, Miller MA, Thacker HL, Snyder PW. Mesial temporal lobe epilepsy: pathogenesis, 
induced rodent models and lesions. Toxicologic pathology 35, 984-999 (2007).

60. Schindler KA, Bialonski S, Horstmann M-T, Elger CE, Lehnertz K. Evolving functional network properties and synchronizability during human epileptic seizures. Chaos: An Interdisciplinary Journal of Nonlinear Science 18, 033119 (2008).

61. Fornito A, Zalesky A, Bullmore E. Fundamentals of brain network analysis. Academic Press (2016).

62. Blumenfeld H. From molecules to networks: cortical/subcortical interactions in the pathophysiology of idiopathic generalized epilepsy. Epilepsia 44 Suppl 2, 7-15 (2003).

63. Vergnes M, Marescaux C, Depaulis A, Micheletti G, Warter JM. Spontaneous spike-and-wave discharges in Wistar rats: a model of genetic generalized nonconvulsive epilepsy. In: Generalized epilepsy). Springer (1990).

64. Danober L, Deransart C, Depaulis A, Vergnes M, Marescaux C. Pathophysiological mechanisms of genetic absence epilepsy in the rat. Progress in neurobiology 55, 27-57 (1998).

65. Gotman J, Grova C, Bagshaw A, Kobayashi E, Aghakhani Y, Dubeau F. Generalized epileptic discharges show thalamocortical activation and suspension of the default state of the brain. Proceedings of the National Academy of Sciences 102, 15236-15240 (2005).

66. Tondelli M, Vaudano AE, Ruggieri A, Meletti S. Cortical and subcortical brain alterations in juvenile absence epilepsy. NeuroImage: Clinical 12, 306-311 (2016).

67. Whelan CD, et al. Structural brain abnormalities in the common epilepsies assessed in a worldwide ENIGMA study. Brain 141, 391-408 (2018).

68. Caciagli L, et al. Abnormal hippocampal structure and function in juvenile myoclonic epilepsy and unaffected siblings. Brain 142, 2670-2687 (2019).

69. Marten F, Rodrigues S, Benjamin O, Richardson MP, Terry JR. Onset of polyspike complexes in a mean-field model of human electroencephalography and its application to absence epilepsy. Philosophical Transactions of the Royal Society A: Mathematical, Physical and Engineering Sciences 367, 11451161 (2009).

70. Bernasconi A, Bernasconi N, Natsume J, Antel S, Andermann F, Arnold D. Magnetic resonance spectroscopy and imaging of the thalamus in idiopathic generalized epilepsy. Brain 126, 2447-2454 (2003).

71. McGill ML, et al. Functional neuroimaging abnormalities in idiopathic generalized epilepsy. NeuroImage: Clinical 6, 455462 (2014).

72. Pfisterer U, et al. Identification of epilepsy-associated neuronal subtypes and gene expression underlying epileptogenesis. Nature communications 11, 1-19 (2020).

73. Aronica E, Gorter JA. Gene expression profile in temporal lobe epilepsy. The Neuroscientist 13, 100-108 (2007).

74. Lee T-S, et al. Gene expression in temporal lobe epilepsy is consistent with increased release of glutamate by astrocytes. Molecular medicine 13, 1-13 (2007).

75. Magalhães PH, Moraes HT, Athie MC, Secolin R, LopesCendes I. New avenues in molecular genetics for the diagnosis and application of therapeutics to the epilepsies. Epilepsy \& Behavior 121, 106428 (2021).

76. Van den Heuvel OA, et al. An overview of the first 5 years of the ENIGMA obsessive-compulsive disorder working group: The power of worldwide collaboration. Human brain mapping, (2020).
77. Ching CR, et al. What we learn about bipolar disorder from large-scale neuroimaging: Findings and future directions from the ENIGMA Bipolar Disorder Working Group. Human brain mapping, (2020).

78. Hoogman $\mathrm{M}$, et al. Consortium neuroscience of attention deficit/hyperactivity disorder and autism spectrum disorder: The ENIGMA adventure. Human brain mapping, (2020).

79. Bas-Hoogendam JM, et al. ENIGMA-anxiety working group: Rationale for and organization of large-scale neuroimaging studies of anxiety disorders. Human Brain Mapping, (2020).

80. Ho TC, et al. Subcortical shape alterations in major depressive disorder: Findings from the ENIGMA major depressive disorder working group. Human brain mapping, (2020).

81. Dale AM, Fischl B, Sereno MI. Cortical surface-based analysis. I. Segmentation and surface reconstruction. Neuroimage $\mathbf{9}$, 179-194 (1999).

82. Achard S, Bullmore E. Efficiency and cost of economical brain functional networks. PLoS Comput Biol 3, e17 (2007).

83. He Y, Chen Z, Evans A. Structural insights into aberrant topological patterns of large-scale cortical networks in Alzheimer's disease. Journal of Neuroscience 28, 4756-4766 (2008).

84. Latora V, Marchiori M. Efficient behavior of small-world networks. Physical review letters 87, 198701 (2001).

85. Bernhardt BC, Bonilha L, Gross DW. Network analysis for a network disorder: the emerging role of graph theory in the study of epilepsy. Epilepsy \& Behavior 50, 162-170 (2015).

86. Markello R, Shafiei G, Y.-Q. Z, Misic B. abagen: A toolbox for the Allen Brain Atlas genetics data. Zenodo, (2020).

87. Arnatkeviciute A, Fulcher BD, Fornito A. A practical guide to linking brain-wide gene expression and neuroimaging data. Neuroimage 189, 353-367 (2019). 
bioRxiv preprint doi: https://doi.org/10.1101/2021.10.18.464713; this version posted October 19,2021 . The copyright holder for this preprint (which was not certified by peer review) is the author/funder, who has granted bioRxiv a license to display the preprint in perpetuity. It is made available under aCC-BY-NC-ND 4.0 International license.

\title{
Structural network alterations in focal and generalized epilepsy follow axes of epilepsy risk gene expression: An ENIGMA study
}

\author{
Supplementary Information
}


bioRxiv preprint doi: https://doi.org/10.1101/2021.10.18.464713; this version posted October 19, 2021 . The copyright holder for this preprint (which was not certified by peer review) is the author/funder, who has granted bioRxiv a license to display the preprint in perpetuity. It is made available under aCC-BY-NC-ND 4.0 International license.

TABLE S1. Site-specific group demographics. Site-specific demographic breakdown of patient-specific subcohorts, including age (in years), age at onset of epilepsy (in years), sex, side of seizure focus (TLE patients only), and mean duration of illness (in years). TLE=Temporal lobe epilepsy, IGE=idiopathic/genetic generalized epilepsy, $\mathrm{HC}=$ healthy controls.

\begin{tabular}{|c|c|c|c|c|c|c|c|}
\hline \multirow{4}{*}{$\begin{array}{c}\begin{array}{c}\text { Site } \\
\text { name }\end{array} \\
\text { BERN }\end{array}$} & \multicolumn{2}{|c|}{$\begin{array}{l}\text { Case-controls } \\
\text { subcohorts }\end{array}$} & \multirow{2}{*}{$\begin{array}{c}\begin{array}{c}\text { Age } \\
(\text { mean } \pm \text { SD })\end{array} \\
31.28 \pm 9.09\end{array}$} & \multirow{2}{*}{$\begin{array}{c}\text { Age at onset } \\
\text { (mean } \pm \text { SD) }\end{array}$} & \multirow{2}{*}{$\begin{array}{c}\begin{array}{c}\text { Sex } \\
\text { (male/female) }\end{array} \\
9 / 9\end{array}$} & \multirow{2}{*}{$\begin{array}{c}\begin{array}{c}\text { Side of focus } \\
\text { (L/R) }\end{array} \\
10 / 8\end{array}$} & \multirow{2}{*}{$\begin{array}{c}\begin{array}{c}\text { Duration } \\
\text { of illness } \\
\text { (mean } \pm \text { SD) }\end{array} \\
-\end{array}$} \\
\hline & TLE & $n=18$ & & & & & \\
\hline & IGE & $n=11$ & $31.36 \pm 8.79$ & - & $5 / 6$ & - & - \\
\hline & $\mathrm{HC}$ & $n=72$ & $31.43 \pm 8.44$ & - & $34 / 38$ & - & - \\
\hline \multirow{3}{*}{ BonN } & TLE & $n=79$ & $34.82 \pm 9.55$ & $15.24 \pm 10.70$ & $37 / 42$ & $55 / 24$ & $19.58 \pm 12.37$ \\
\hline & IGE & $n=0$ & - & - & - & - & - \\
\hline & $\mathrm{HC}$ & $n=57$ & $34.09 \pm 969$ & - & $30 / 27$ & - & - \\
\hline \multirow{3}{*}{ BRUSSELS } & TLE & $n=13$ & $35.85 \pm 5.27$ & $12.50 \pm 10.60$ & $5 / 8$ & $10 / 3$ & $23.25 \pm 13.21$ \\
\hline & IGE & $n=0$ & - & - & - & - & - \\
\hline & $\mathrm{HC}$ & $n=44$ & $26.64 \pm 4.34$ & - & $20 / 24$ & - & - \\
\hline \multirow{3}{*}{ CUBRIC } & TLE & $n=0$ & - & - & - & - & - \\
\hline & IGE & $n=44$ & $27.61 \pm 7.39$ & $12.95 \pm 4.67$ & $12 / 32$ & - & $14.61 \pm 10.03$ \\
\hline & $\mathrm{HC}$ & $n=48$ & $28.04 \pm 8.17$ & - & $14 / 34$ & - & - \\
\hline \multirow{3}{*}{ EPICZ } & TLE & $n=40$ & $37.73 \pm 8.06$ & $16.50 \pm 13.20$ & $18 / 22$ & $17 / 23$ & $21.23 \pm 13.00$ \\
\hline & IGE & $n=0$ & - & - & - & - & - \\
\hline & $\mathrm{HC}$ & $n=96$ & $35.58 \pm 8.80$ & - & $47 / 49$ & - & - \\
\hline \multirow{3}{*}{ EPIGEN_3.0 } & TLE & $n=13$ & $40.39 \pm 6.28$ & $21.84 \pm 13.16$ & $7 / 6$ & $8 / 5$ & $18.55 \pm 11.98$ \\
\hline & IGE & $n=0$ & - & - & - & - & - \\
\hline & $\mathrm{HC}$ & $n=64$ & $33.11 \pm 8.00$ & - & $35 / 29$ & - & - \\
\hline \multirow{3}{*}{ GREIFSWALD } & TLE & $n=0$ & - & - & - & - & - \\
\hline & IGE & $n=25$ & $32.56 \pm 7.85$ & $19.44 \pm 9.73$ & $12 / 13$ & - & $13.12 \pm 9.96$ \\
\hline & $\mathrm{HC}$ & $n=96$ & $25.76 \pm 5.00$ & - & $36 / 60$ & - & - \\
\hline \multirow{3}{*}{ IDIBAPS-HCP } & TLE & $n=46$ & $35.02 \pm 8.43$ & $15.22 \pm 11.28$ & $21 / 25$ & $14 / 32$ & $18.97 \pm 10.35$ \\
\hline & IGE & $n=3$ & $38.33 \pm 3.51$ & - & $2 / 1$ & - & - \\
\hline & $\mathrm{HC}$ & $n=51$ & $32.75 \pm 5.35$ & - & $23 / 28$ & - & - \\
\hline \multirow{3}{*}{ KCL_CNS } & TLE & $n=13$ & $38.85 \pm 8.32$ & $16.73 \pm 13.34$ & $4 / 9$ & $5 / 8$ & $22.91 \pm 15.80$ \\
\hline & IGE & $n=31$ & $29.94 \pm 8.42$ & $12.00 \pm 5.73$ & $11 / 20$ & - & $17.94 \pm 8.93$ \\
\hline & $\mathrm{HC}$ & $n=97$ & $30.82 \pm 7.39$ & - & $45 / 52$ & - & - \\
\hline \multirow{3}{*}{ KUOPIO } & TLE & $n=0$ & - & - & - & - & - \\
\hline & IGE & $n=35$ & $27.86 \pm 8.34$ & $17.46 \pm 9.93$ & $13 / 22$ & - & $10.46 \pm 10.67$ \\
\hline & $\mathrm{HC}$ & $n=67$ & $25.16 \pm 1.55$ & - & $34 / 33$ & - & - \\
\hline \multirow{3}{*}{ MNI } & TLE & $n=79$ & $33.10 \pm 8.82$ & $17.09 \pm 10.08$ & $34 / 45$ & $44 / 35$ & $16.01 \pm 10.72$ \\
\hline & IGE & $n=0$ & - & - & - & - & - \\
\hline & $\mathrm{HC}$ & $n=45$ & $30.24 \pm 6.65$ & - & $25 / 20$ & - & - \\
\hline \multirow{3}{*}{ NYU } & TLE & $n=17$ & $31.76 \pm 7.34$ & $15.12 \pm 7.74$ & $7 / 10$ & $7 / 10$ & $17.12 \pm 11.68$ \\
\hline & IGE & $n=35$ & $32.43 \pm 9.34$ & $14.23 \pm 5.25$ & $19 / 16$ & - & $18.38 \pm 9.86$ \\
\hline & $\mathrm{HC}$ & $n=108$ & $28.51 \pm 8.88$ & - & $52 / 56$ & - & - \\
\hline \multirow{3}{*}{ RMH } & TLE & $n=25$ & $34.80 \pm 9.22$ & $23.24 \pm 12.72$ & $15 / 10$ & $16 / 9$ & $12.03 \pm 12.51$ \\
\hline & IGE & $n=21$ & $31.33 \pm 8.79$ & $22.28 \pm 9.43$ & $8 / 13$ & - & $9.51 \pm 13.81$ \\
\hline & $\mathrm{HC}$ & $n=19$ & $29.21 \pm 8.59$ & - & $12 / 7$ & - & - \\
\hline
\end{tabular}


bioRxiv preprint doi: https://doi.org/10.1101/2021.10.18.464713; this version posted October $19,2021$. The copyright holder for this preprint (which was not certified by peer review) is the author/funder, who has granted bioRxiv a license to display the preprint in perpetuity. It is made available under aCC-BY-NC-ND 4.0 International license.

Larivière et al. Imaging-genetic associations in the common epilepsies | 17

\begin{tabular}{|c|c|c|c|c|c|c|c|}
\hline $\begin{array}{c}\text { Site } \\
\text { name }\end{array}$ & \multicolumn{2}{|c|}{$\begin{array}{c}\text { Case-controls } \\
\text { subcohorts }\end{array}$} & $\begin{array}{c}\text { Age } \\
(\text { mean } \pm \text { SD })\end{array}$ & $\begin{array}{c}\text { Age at onset } \\
(\text { mean } \pm \text { SD) }\end{array}$ & $\begin{array}{c}\text { Sex } \\
\text { (male/female) }\end{array}$ & $\begin{array}{c}\text { Side of focus } \\
(L / R)\end{array}$ & $\begin{array}{l}\text { Duration } \\
\text { of illness }\end{array}$ \\
\hline \multirow{3}{*}{ UCSD } & TLE & $n=17$ & $33.24 \pm 8.42$ & $14.87 \pm 12.60$ & $8 / 9$ & $10 / 7$ & $19.53 \pm 13.53$ \\
\hline & IGE & $n=0$ & - & - & - & - & - \\
\hline & $\mathrm{HC}$ & $n=29$ & $30.10 \pm 7.77$ & - & $16 / 13$ & - & - \\
\hline \multirow{3}{*}{ UNAM } & TLE & $n=17$ & $30.76 \pm 9.50$ & $14.53 \pm 12.73$ & $7 / 10$ & $9 / 8$ & $16.18 \pm 9.54$ \\
\hline & IGE & $n=0$ & - & - & - & - & - \\
\hline & $\mathrm{HC}$ & $n=31$ & $31.61 \pm 10.58$ & - & $7 / 24$ & - & - \\
\hline \multirow{3}{*}{ UNICAMP } & TLE & $n=161$ & $40.83 \pm 7.64$ & $11.49 \pm 9.70$ & $70 / 91$ & $91 / 70$ & $29.65 \pm 11.55$ \\
\hline & IGE & $n=37$ & $32.27 \pm 9.31$ & $11.46 \pm 7.22$ & $9 / 28$ & - & $20.81 \pm 10.08$ \\
\hline & $\mathrm{HC}$ & $n=357$ & $32.26 \pm 8.83$ & - & $135 / 222$ & - & - \\
\hline \multirow{3}{*}{ UNIMORE } & TLE & $n=0$ & - & - & - & - & - \\
\hline & IGE & $n=38$ & $24.61 \pm 7.46$ & $11.32 \pm 5.75$ & $14 / 24$ & - & $11.39 \pm 9.86$ \\
\hline & $\mathrm{HC}$ & $n=34$ & $28.47 \pm 5.25$ & - & $14 / 20$ & - & - \\
\hline \multirow{3}{*}{ XMU } & TLE & $n=40$ & $28.25 \pm 8.45$ & $17.18 \pm 12.06$ & $25 / 15$ & $25 / 15$ & $11.28 \pm 8.02$ \\
\hline & IGE & $n=11$ & $31.00 \pm 10.45$ & $18.56 \pm 16.49$ & $7 / 4$ & - & $12.00 \pm 10.23$ \\
\hline & $\mathrm{HC}$ & $n=13$ & $31.54 \pm 6.99$ & - & $9 / 4$ & - & - \\
\hline
\end{tabular}


bioRxiv preprint doi: https://doi.org/10.1101/2021.10.18.464713; this version posted October 19, 2021. The copyright holder for this preprint (which was not certified by peer review) is the author/funder, who has granted bioRxiv a license to display the preprint in perpetuity. It is made available under aCC-BY-NC-ND 4.0 International license.

TABLE S2. List of disease-specific risk genes from previously published GWAS.

\begin{tabular}{|c|c|c|}
\hline Disorder & Genes & Ref \\
\hline All epilepsies & $\begin{array}{l}\text { ATXN1, BCL11A, BRD7, C3orf33, FANCL, GABRA2, GJA1, } \\
\text { GRIK1, HEATR3, KCNAB1, KCNN2, PCDH7, PNPO, SCN1A, } \\
\text { SCN2A, SCN3A, STAT4, STX1B, TTC21B, ZEB2 }\end{array}$ & 32 \\
\hline $\begin{array}{l}\text { Focal epilepsy } \\
\text { (hippocampal } \\
\text { sclerosis) }\end{array}$ & C3orf33, GJA1, KCNAB1 & 32 \\
\hline Generalized epilepsy & $\begin{array}{c}\text { ATXN1, BCL11A, FANCL, GABRA2, GRIK1, KCNN2, PCDH7, } \\
\text { PNPO, SCN1A, SCN2A, SCN3A, STAT4, TTC21B }\end{array}$ & 32 \\
\hline $\begin{array}{l}\text { Attention deficit/ } \\
\text { hyperactivity disorder }\end{array}$ & $\begin{array}{l}\text { ARTN, B4GALT2, CCDC24, DPH2, DUSP6, FOXP2, IPO13, } \\
\text { KDM4A, LINC00461, PCDH7, POC1B, PTPRF, SEMA6D, } \\
\text { SORCS3, SPAG16, ST3GAL3, TMEM161B_AS1 }\end{array}$ & 41 \\
\hline $\begin{array}{l}\text { Autism spectrum } \\
\text { disorder }\end{array}$ & $\begin{array}{l}\text { APOPT1, BAG5, C8orf74, CADPS, CKB, FEZF2, KCNN2, KIZ, } \\
\text { KLC1, KMT2E, MACROD2, MROH5, NEGR1, NKX2_2, } \\
\text { NUDT12, PINX1, POU3F2, PTBP2, SOX7, TMEM33, TRMT61A, } \\
\text { XRN2 }\end{array}$ & 42 \\
\hline Bipolar disorder & $\begin{array}{l}\text { ADCY2, ADD3, ANK3, CACNA1C, CD47, FADS2, FSTL5, } \\
\text { GRIN2A, HDAC5, LMAN2L, MRPS33, NCAN, PACS1, PC, } \\
\text { PLEKHO1, POU3F2, RIMS1, RPS6KA2, SCN2A, SHANK2, } \\
\text { SSBP2, STARD9, STK4, THSD7A, TRANK1, ZCCHC2, ZNF592 }\end{array}$ & 43 \\
\hline $\begin{array}{l}\text { Major depressive } \\
\text { disorder }\end{array}$ & $\begin{array}{l}\text { ABT1, ACVR1B, ANKHD1, ANKK1, ANKS1B, AP3B1, APOPT1, } \\
\text { ARHGEF25, ASCC3, ASIC2, ASTN2, ASXL3, ATP1A3, BAD, } \\
\text { BAG5, BAZ2B, BCHE, BSN, BTN2A1, BTN3A2, BTN3A3, } \\
\text { C16orf45, CABP1, CACNA1E, CACNA2D1, CAMKK2, CDH13, } \\
\text { CDH22, CDH9, CDK14, CELF2, CELF4, CHD6, CKB, CNTN5, } \\
\text { CNTNAP5, CRB1, CSMD1, CTNNA3, CTTNBP2, DCC, DCDC1, } \\
\text { DENND1A, DENND1B, DLST, DRD2, ELAVL2, EMILIN3, } \\
\text { EPHB2, ERBB4, ESRRG, ETFDH, EXT1, FADS1, FADS2, } \\
\text { FAM120A, FAM120AOS, FAM172A, FANCL, FCF1, FH, FHIT, } \\
\text { FNIP2, GINM1, GPC5, GPC6, GRIK5, GRM5, GRM8, GTF2IRD1, } \\
\text { HARS, HIST1H2BF, HIST1H2BL, HIST1H2BN, HIST1H2BO, } \\
\text { HS6ST3, HSPA1A, HTT, IGSF6, IK, INPP4B, KDM3A, KIAA1143, } \\
\text { KLC1, KMT2A, KYNU, LIN28B, LPIN3, LRFN5, LRP1B, LST1, } \\
\text { LTBP3, MANEA, MAP9, MED19, MEF2C, MEGF11, METTL9, } \\
\text { MGAT4C, MICB, MIER1, MR1, MYBPC3, MYRF, NEGR1, } \\
\text { NICN1, NRG1, OLFM4, PAX6, PCDH9, PCDHA1, PCDHA5, } \\
\text { PCLO, PCNP, PGBD1, PLA2R1, PLCL1, PMFBP1, POGZ, PPID, } \\
\text { PPP6C, PRR16, PRSS16, PSEN2, PSORS1C1, PTPRS, RAB27B, } \\
\text { RAB3B, RABEPK, RANGAP1, RBFOX1, RBMS3, RFTN2, RHOA, } \\
\text { RHOBTB1, RPS6KL1, RSRC1, RTN1, SAMD5, SCAI, SCYL1, } \\
\text { SDK1, SEMA6D, SERPING1, SF3B1, SGIP1, SHISA9, SLC4A9, } \\
\text { SORBS3, SORCS3, SOX5, SOX6, SPPL3, SPRY2, STAU1, STK19, } \\
\text { TAL1, TCAIM, TCF4, TCTEX1D1, TENM2, TMEM106B, } \\
\text { TMEM161B, TMEM258, TMEM42, TMEM67, TRAF3, TRMT10C, } \\
\text { TRMT61A, TTC12, UBE2M, USP3, VPS41, VRK2, YLPM1, } \\
\text { ZC3H7B, ZCCHC7, ZDHHC21, ZDHHC5, ZFHX4, ZHX3, } \\
\text { ZKSCAN4, ZKSCAN8, ZMAT2, ZNF165, ZNF184, ZNF322, } \\
\text { ZNF35, ZNF536, ZNF638, ZNF660, ZSCAN26, ZSCAN31, } \\
\text { ZSCAN9 }\end{array}$ & 44 \\
\hline
\end{tabular}


bioRxiv preprint doi: https://doi.org/10.1101/2021.10.18.464713; this version posted October 19, 2021. The copyright holder for this preprint (which was not certified by peer review) is the author/funder, who has granted bioRxiv a license to display the preprint in perpetuity. It is made available under aCC-BY-NC-ND 4.0 International license.

Schizophrenia

ABCB9, ACTR5, ADAMTSL3, ALDOA, AMBRA1, ANKRD44, ANP32E, APH1A, APOPT1, ARHGAP1, ARL3, ARL6IP4, ASPHD1, ATG13, ATP2A2, BAG5, C12orf65, C1 orf54, C2orf69, CA14, CACNA1C, CACNA1I, CACNB2, CCDC39, CDK2AP1, CENPM, CHADL, CHRM4, CHRNA3, CILP2, CKAP5, CKB, CLCN3, CNKSR2, CNNM2, CNTN4, DGKZ, DNAJC19, DOC2A, DPYD, DRD2, EFHD1, ERCC4, ESAM, FAM57B, FANCL, FES, FUT9, FXR1, GATAD2A, GIGYF2, GNL3, GRM3, HAPLN4, HARBI1, HIRIP3, HSPD1, HSPE1, IMMP2L, INA, IREB2, KCNV1, KCTD13, KDM4A, KLC1, L3MBTL2, LRP1, MAD1L1, MAN2A2, MAPK3, MDK, MPHOSPH9, MSANTD2, MSL2, NAB2, NAGA, NCAN, NCK1, NDUFA13, NDUFA4L2, NDUFA6, NEK1, NEK4, NGEF, NISCH, NRGN, NT5C2, NT5DC2, NXPH4, OGFOD2, OTUD7B, PBRM1, PBX4, PCCB, PCGF6, PITPNM2, PJA1, PLCH2, PLCL1, PLEKHO1, PPP1R13B, PPP1R16B, PPP2R3A, PTPRF, PUS7, R3HDM2, RANGAP1, RFTN2, RILPL2, SBNO1, SEPT3, SEZ6L2, SF3B1, SGSM, SHISA8, SHMT2, SLC32A1, SLC35G2, SLC39A8, SLC45A1, SMDT1, SMG6, SMIM4, SNAP91, SNX19, SREBF2, SRR, STAB1, STAG1, STAT6, SUGP1, TAC3, TAF5, TCF20, TCF4, TM6SF2, TMEM219, TNFRSF13C, TRANK1, TRIM8, TRMT61A, TSNARE1, TSR1, TYW5, VRK2, WBP1L, YPEL3, ZFYVE21, ZSCAN2, ZSWIM6 
bioRxiv preprint doi: https://doi.org/10.1101/2021.10.18.464713; this version posted October 19, 2021. The copyright holder for this preprint (which was not certified by peer review) is the author/funder, who has granted bioRxiv a license to display the preprint in perpetuity. It is made available under aCC-BY-NC-ND 4.0 International license.

a Global and regional network alterations in left TLE
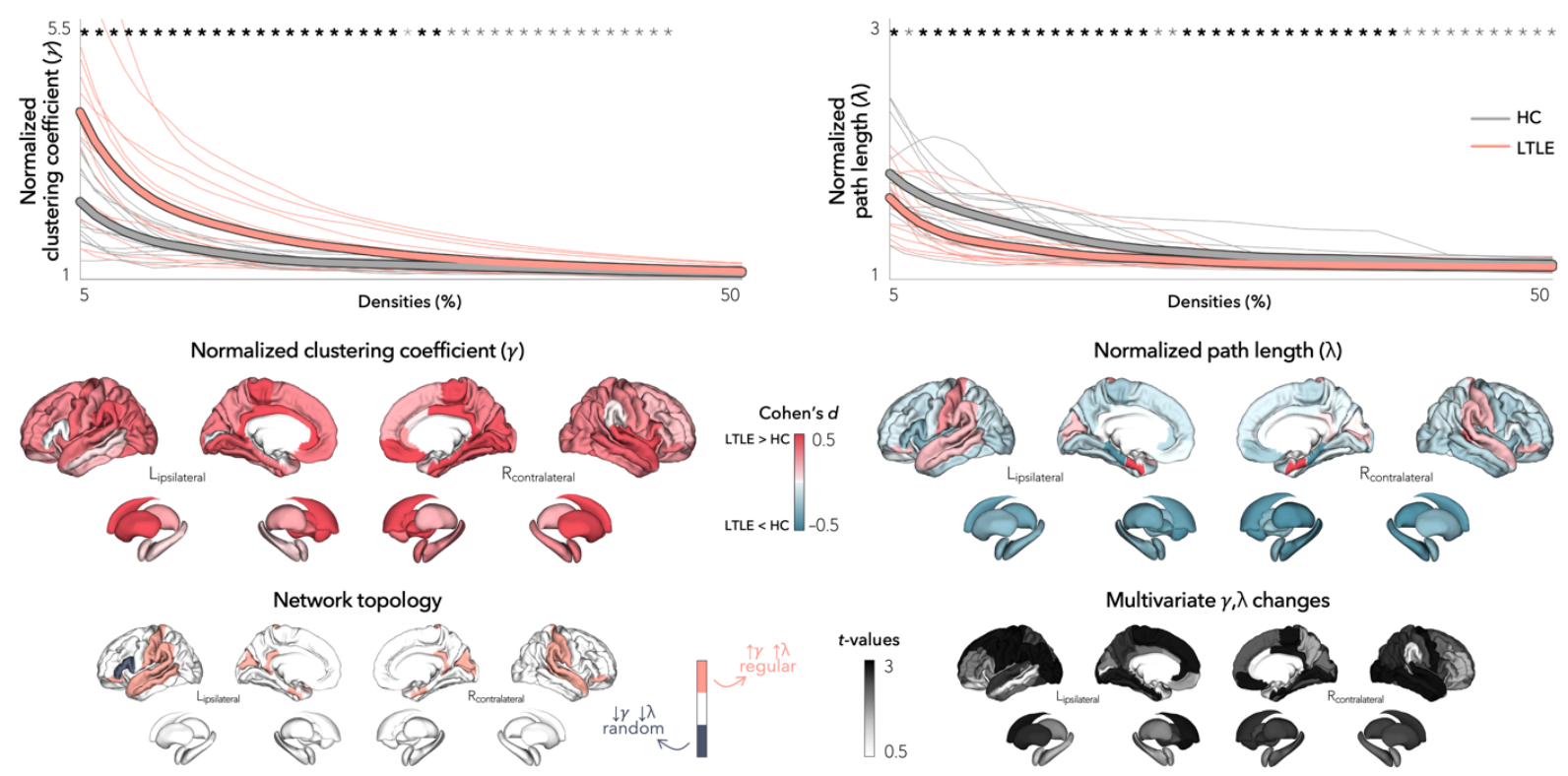

b | Transcriptomic associations
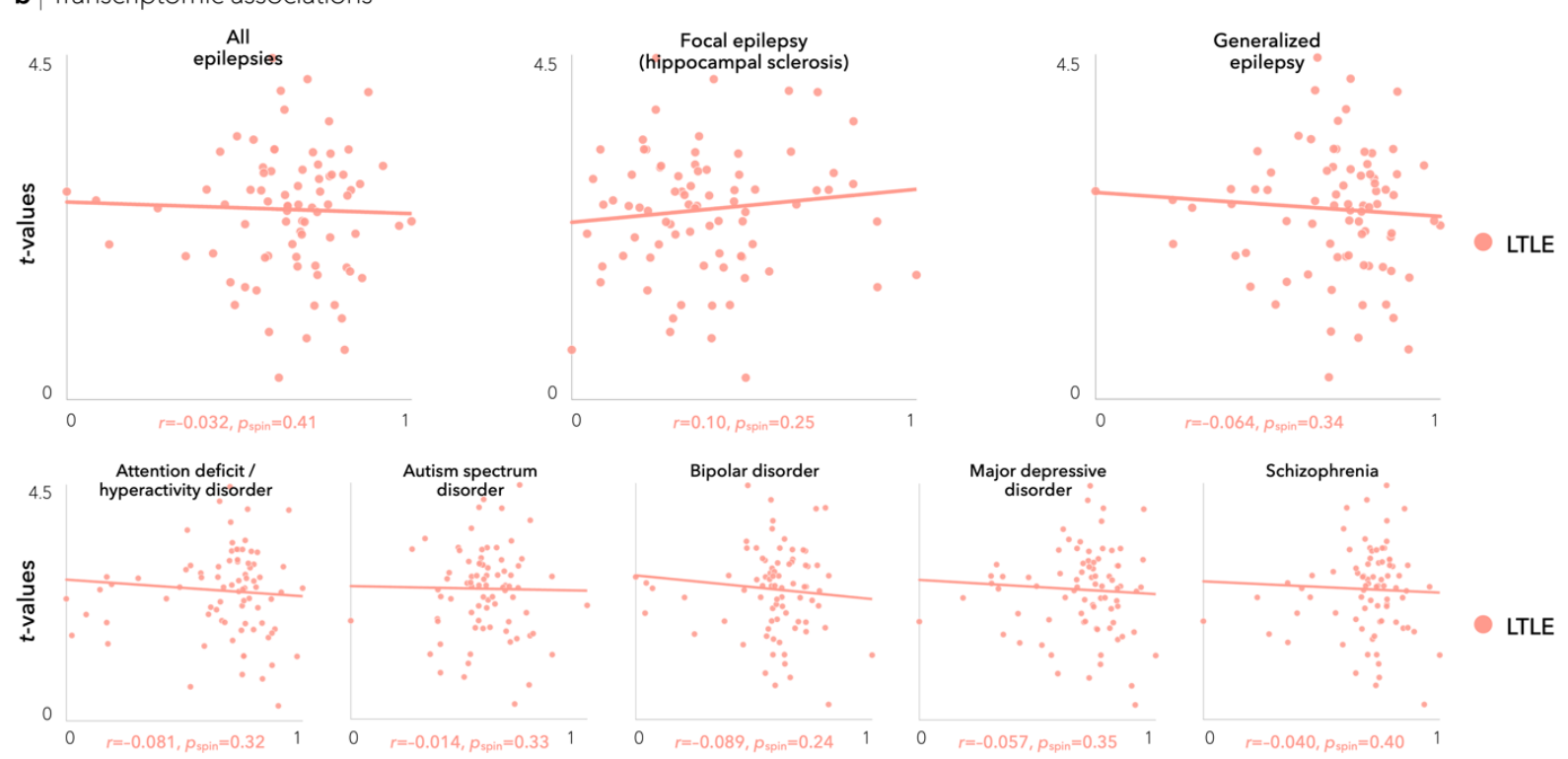

Gene co-expression levels

FIGURE S1. Structural covariance networks in left TLE (LTLE). (a) Global differences in clustering coefficient (top left) and path length (top right) between left LTLE and healthy controls (HC) are plotted as a function of network density. Increased small-worldness (increased clustering coefficient, decreased path length) was observed in individuals with left TLE. Student's t-tests were performed at each density value; bold asterisks indicate $p_{\mathrm{FDR}}<0.05$, semi-transparent asterisks indicate $p_{\mathrm{FDR}}<0.1$. Thin lines represent data from individual sites. Multivariate topological differences in left TLE were primarily observed in bilateral fronto-temporal cortices, and revealed a regular network configuration (increased clustering and path length). (b) Associations between epilepsy-related gene co-expression profiles and multivariate changes were marginal, although correlation to hippocampal sclerosis genes was strongest. 
bioRxiv preprint doi: https://doi.org/10.1101/2021.10.18.464713; this version posted October 19, 2021. The copyright holder for this preprint (which was not certified by peer review) is the author/funder, who has granted bioRxiv a license to display the preprint in perpetuity. It is made available under aCC-BY-NC-ND 4.0 International license.

a | Global and regional network alterations in right TLE
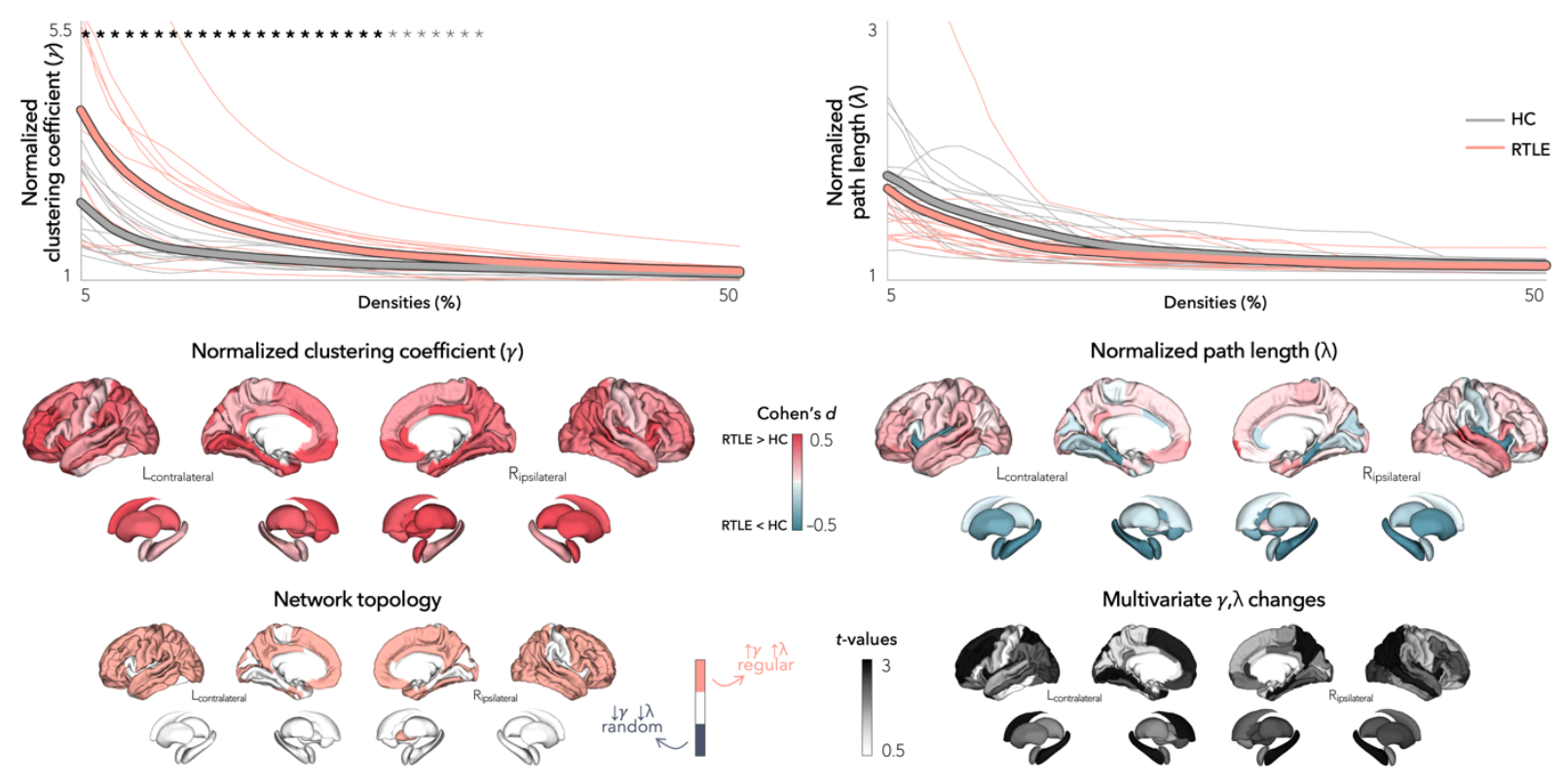

b | Transcriptomic associations
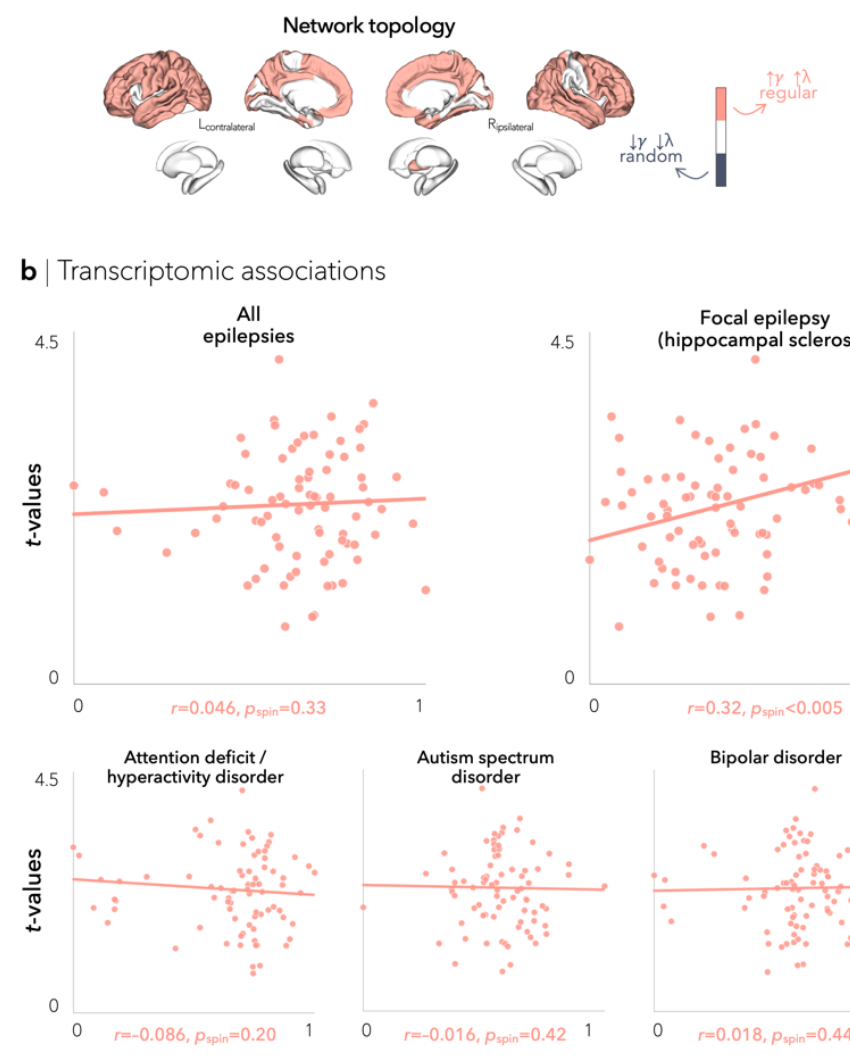

Focal epilepsy
(hippocampal sclerosis)
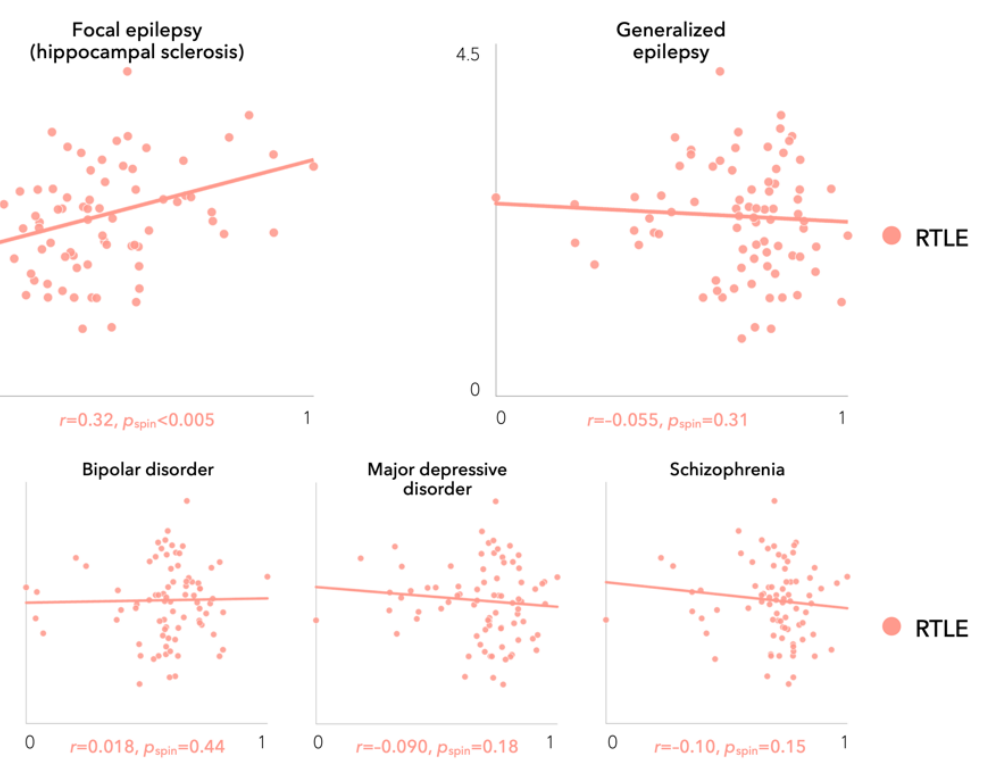

Gene co-expression levels

FIGURE S2. Structural covariance networks in right TLE (RTLE). (a) Global differences in clustering coefficient (top left) and path length (top right) between RTLE and healthy controls (HC) are plotted as a function of network density. Increased small-worldness (increased clustering coefficient, decreased path length) was observed in individuals with right TLE. Student's t-tests were performed at each density value; bold asterisks indicate $p_{\mathrm{FDR}}<0.05$, semi-transparent asterisks indicate $p_{\mathrm{FDR}}<0.1$. Thin lines represent data from individual sites. Multivariate topological changes in right TLE were primarily observed in bilateral fronto-temporal cortices and the hippocampus, and revealed a widespread regular network configuration (increased clustering and path length). (b) Associations between epilepsy-related gene co-expression profiles and multivariate changes were strongest for hippocampal sclerosis genes. 
bioRxiv preprint doi: https://doi.org/10.1101/2021.10.18.464713; this version posted October 19,2021 . The copyright holder for this preprint (which was not certified by peer review) is the author/funder, who has granted bioRxiv a license to display the preprint in perpetuity. It is made available under aCC-BY-NC-ND 4.0 International license.

a | Global network alterations in left vs. right TLE
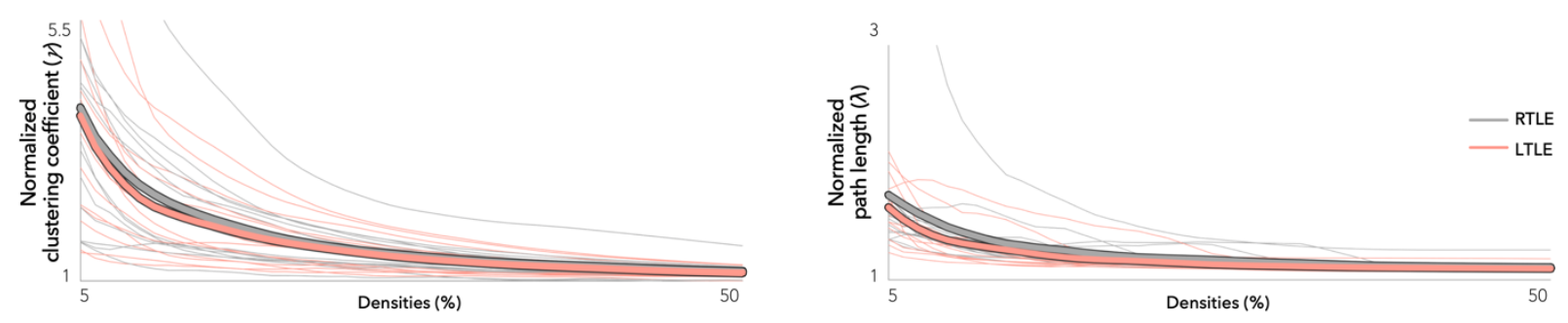

b | Regional network alterations in left vs. right TLE

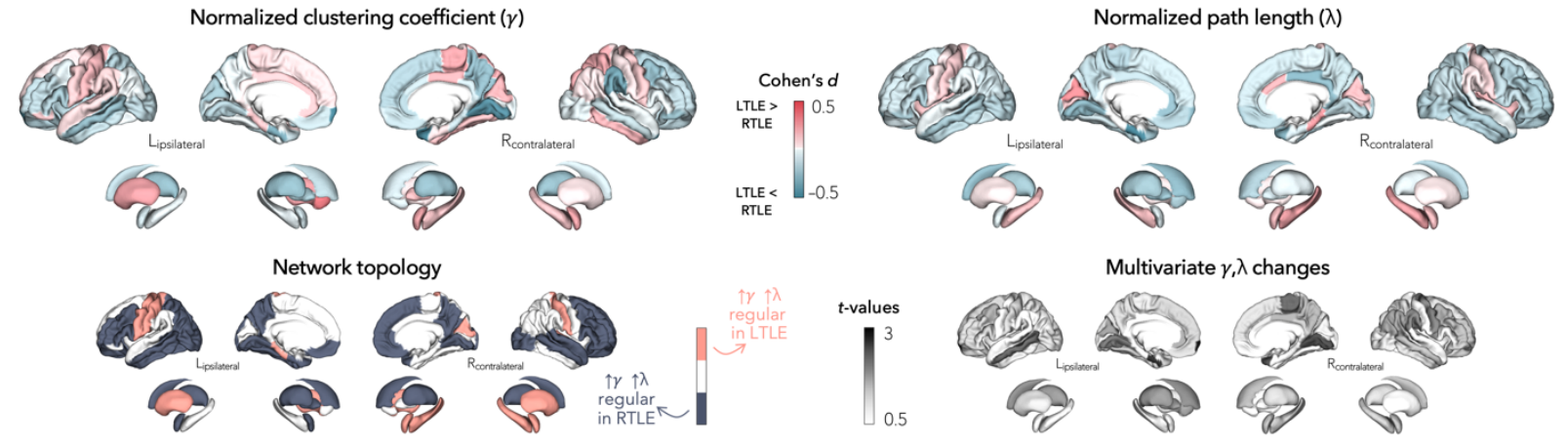

FIGURE S3. Structural covariance networks in left (LTLE) vs. right (RTLE) TLE. (a) Global differences in clustering coefficient (left) and path length (right) between LTLE and RTLE are plotted as a function of network density. No significant difference was observed. Student's t-tests were performed at each density value; bold asterisks indicate $p_{\mathrm{FDR}}<0.05$, semi-transparent asterisks indicate $p_{\mathrm{FDR}}<0.1$. Thin lines represent data from individual sites. (b) Trends for multivariate topological changes in LTLE vs. RTLE were observed in ipsilateral middle frontal gyrus and enthorinal cortex as well as contralateral calcarine sulcus. Compared to the other subcohort, LTLE showed network regularization (increased clustering and path length) in sensorimotor cortices, whereas RTLE showed widespread network regularization. 
bioRxiv preprint doi: https://doi.org/10.1101/2021.10.18.464713; this version posted October 19, 2021. The copyright holder for this preprint (which was not certified by peer review) is the author/funder, who has granted bioRxiv a license to display the preprint in perpetuity. It is made available under aCC-BY-NC-ND 4.0 International license.

a | Global and regional network alterations in patients with short duration of TLE
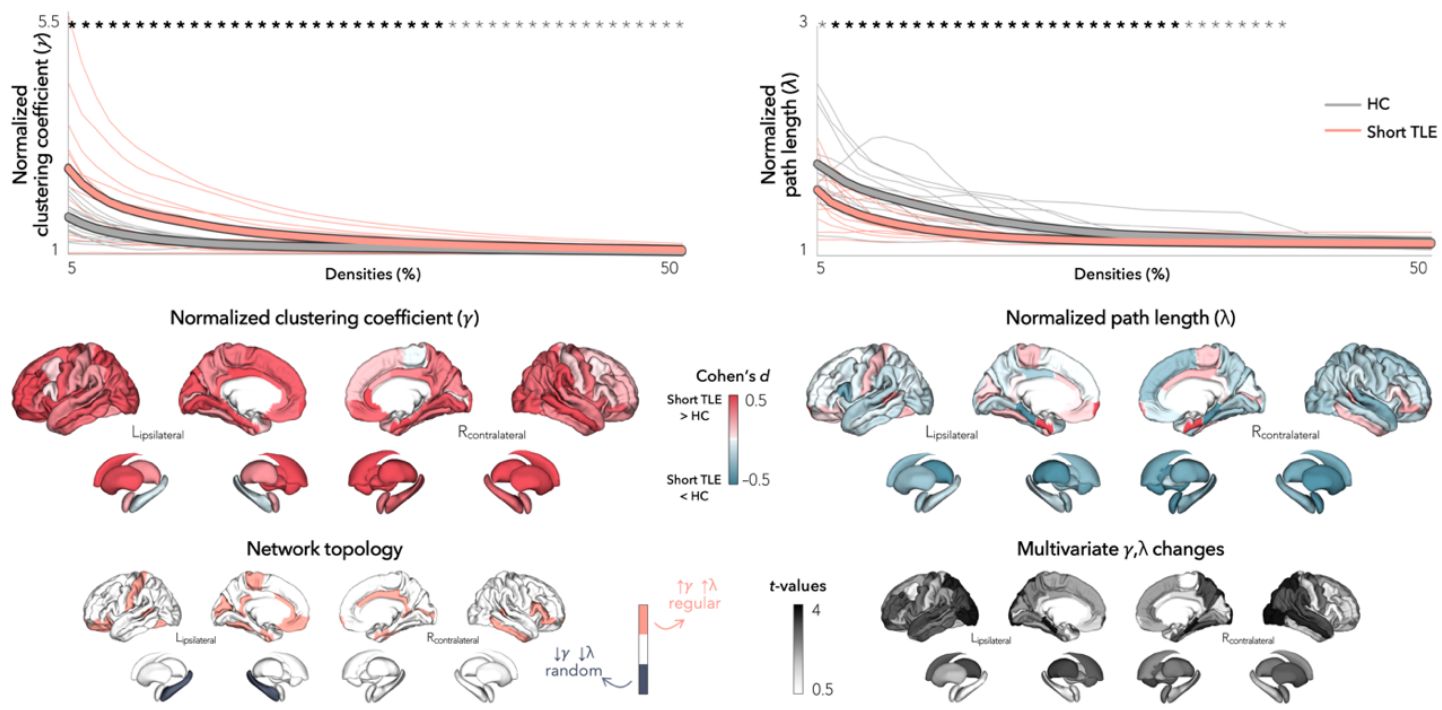

b | Global and regional network alterations in patients with long duration of TLE
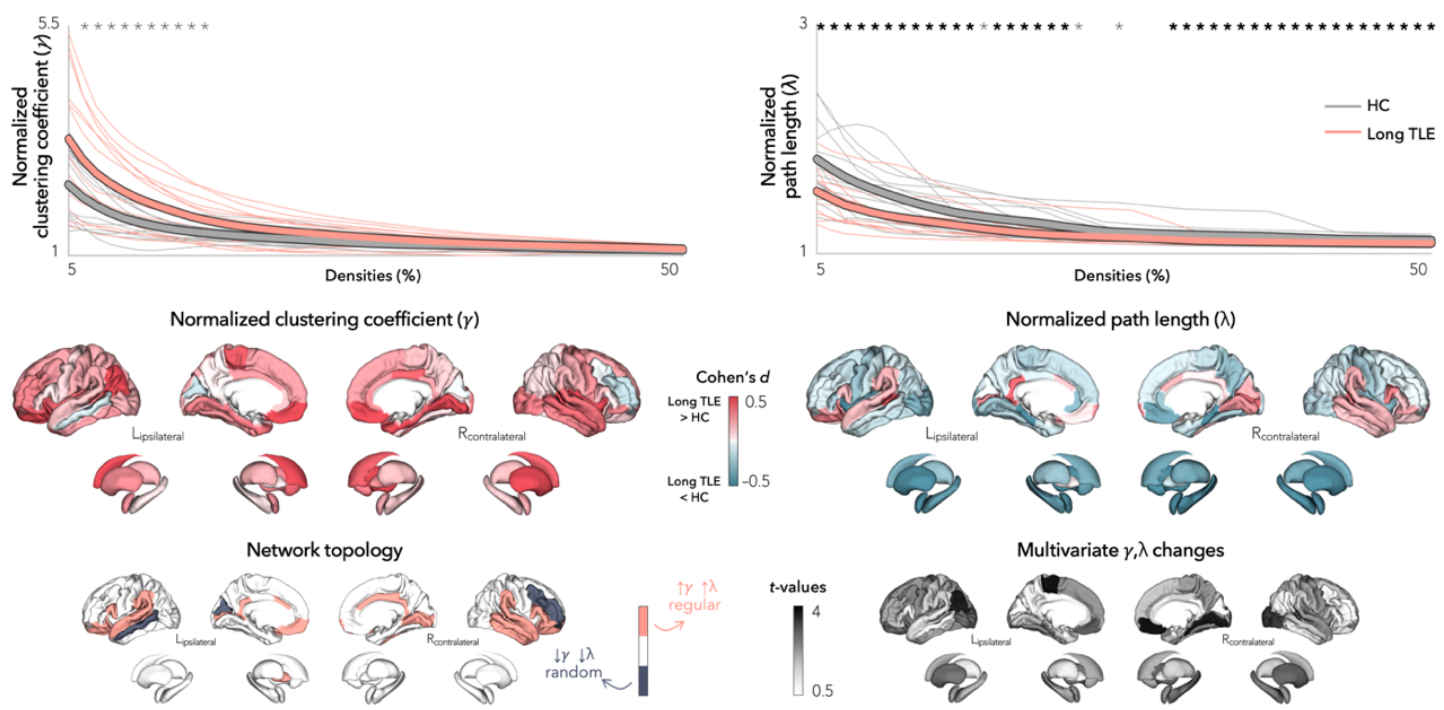

FIGURE S4. Structural covariance networks in patients with short and long TLE duration. (a) Global differences in clustering coefficient (top left) and path length (top right) between Short TLE and healthy controls $(\mathrm{HC})$ are plotted as a function of network density. Increased small-worldness (increased clustering coefficient, decreased path length) was observed in patients with short TLE duration. Student's t-tests were performed at each density value; bold asterisks indicate $p_{\mathrm{FDR}}<0.05$, semi-transparent asterisks indicate $p_{\mathrm{FDR}}<0.1$. Thin lines represent data from individual sites. Multivariate topological changes in patients with short TLE duration were widespread, affecting bilateral fronto-parietal and limbic cortices and the thalamus, and revealed a regular subnetwork configuration (increased clustering and path length). (b) Global differences in clustering coefficient (top left) and path length (top right) between Long TLE and healthy controls $(\mathrm{HC})$ are plotted as a function of network density. Increased small-worldness (increased clustering coefficient, decreased path length) was observed in patients with long TLE duration. Multivariate topological changes in patients with long TLE duration were primarily observed in fronto-limbic cortices and bilateral putamen, amygdala, and hippocampus, and revealed a regular subnetwork configuration (increased clustering and path length). 
bioRxiv preprint doi: https://doi.org/10.1101/2021.10.18.464713; this version posted October 19, 2021. The copyright holder for this preprint (which was not certified by peer review) is the author/funder, who has granted bioRxiv a license to display the preprint in perpetuity. It is made available under aCC-BY-NC-ND 4.0 International license.

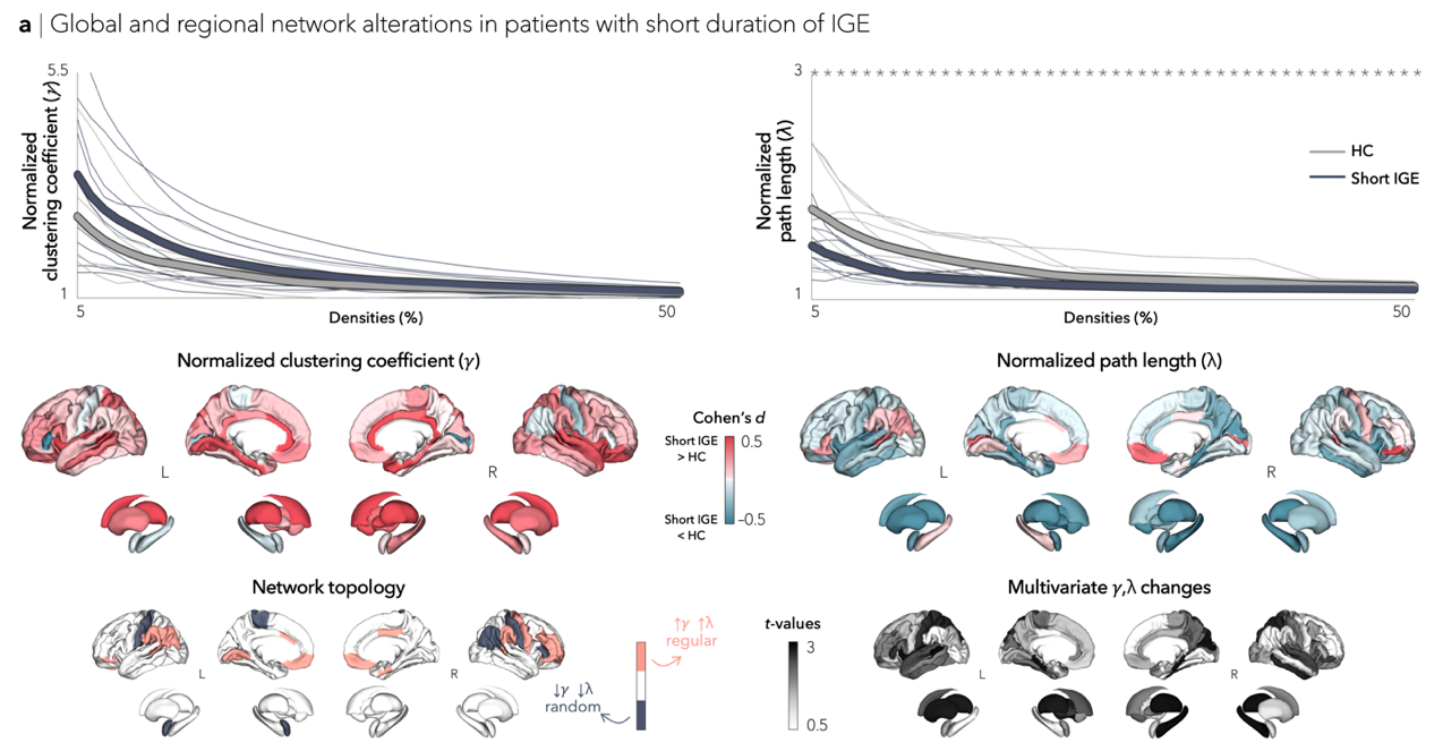

b | Global and regional network alterations in patients with long duration of IGE
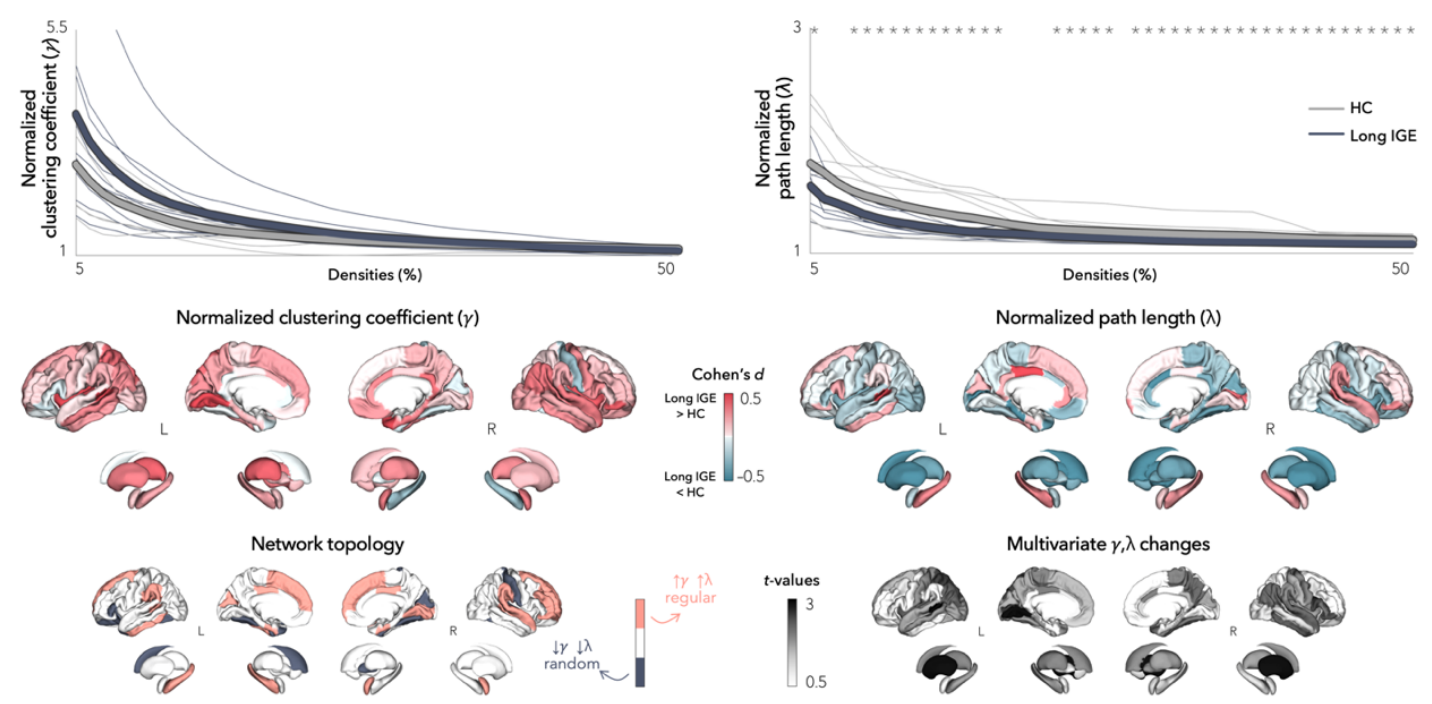

Normalized path length $(\lambda)$

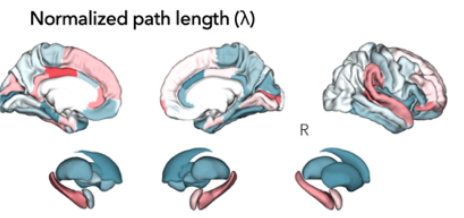

FIGURE S5. Structural covariance networks in patients with short and long IGE duration. (a) Global differences in clustering coefficient (top left) and path length (top right) between Short IGE and healthy controls $(\mathrm{HC})$ are plotted as a function of network density. Decreased path length was observed in patients with short IGE duration. Student's t-tests were performed at each density value; bold asterisks indicate $p_{\mathrm{FDR}}<0.05$, semi-transparent asterisks indicate $p_{\mathrm{FDR}}<0.1$. Thin lines represent data from individual sites. Multivariate topological changes in patients with short IGE duration were observed, primarily in bilateral fronto-central and temporal cortices, as well as bilateral thalamus and right hippocampus. Both random (decreased clustering and path length in centro-parietal regions) and regular (increased clustering and path length in orbitofrontal and parietal regions) network configurations were observed. (b) Global differences in clustering coefficient (top left) and path length (top right) between Long IGE and healthy controls (HC) are plotted as a function of network density. Decreased path length was observed in patients with long IGE duration. Multivariate topological changes in patients with long IGE duration were primarily observed in bilateral fronto-temporo-parietal regions and bilateral putamen. Once again, both random (fronto-central cortices) and regular (fronto-temporo-parietal regions) network configurations were observed. 
bioRxiv preprint doi: https://doi.org/10.1101/2021.10.18.464713; this version posted October 19, 2021. The copyright holder for this preprint (which was not certified by peer review) is the author/funder, who has granted bioRxiv a license to display the preprint in perpetuity. It is made available under aCC-BY-NC-ND 4.0 International license.
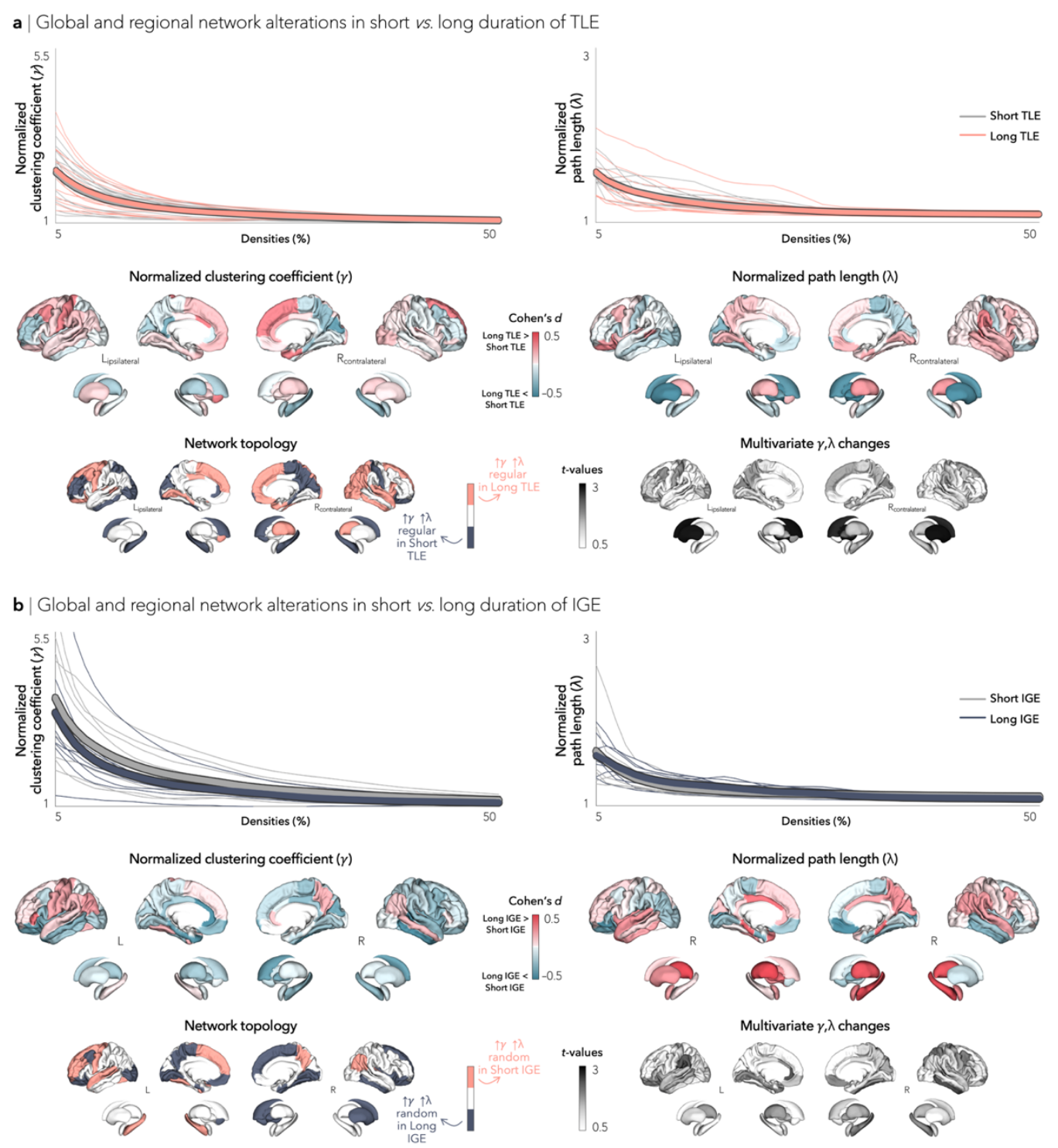

FIGURE S6. Structural covariance networks in short vs. long TLE/IGE duration. (a) Global differences in clustering coefficient (top left) and path length (top right) between Short and Long TLE are plotted as a function of network density; no significant difference was observed in clustering coefficient or path length. Student's t-tests were performed at each density value; bold asterisks indicate $p_{F D R}<0.05$, semi-transparent asterisks indicate $p_{\mathrm{FDR}}<0.1$. Thin lines represent data from individual sites. Marginal multivariate topological changes were observed between groups, primarily affecting bilateral caudate and ipsilateral putamen. Patients with shorter duration exhibited network regularization (increased clustering and path length) in bilateral fronto-parietal cortices and hippocampus, whereas patients with longer duration exhibited network regularization more broadly in fronto-temporo-parietal cortices. (b) Global differences in clustering coefficient (top left) and path length (top right) between Short and Long IGE are plotted as a function of network density; no significant difference was observed in clustering coefficient or path length. Marginal multivariate topological changes were observed between groups, primarily affecting bilateral fronto-limbic cortices. Patients with shorter duration exhibited network randomization (decreased clustering and path length) in left fronto-temporal cortices and hippocampus and right parietal cortices, whereas patients with longer duration exhibited network randomization in left fronto-parieto-occipital cortices and right fronto-temporal cortices, caudate, and putamen. 
bioRxiv preprint doi: https://doi.org/10.1101/2021.10.18.464713; this version posted October 19,2021 . The copyright holder for this preprint (which was not certified by peer review) is the author/funder, who has granted bioRxiv a license to display the preprint in perpetuity. It is made available under aCC-BY-NC-ND 4.0 International license.

a | Topological network regularization in TLE across sites
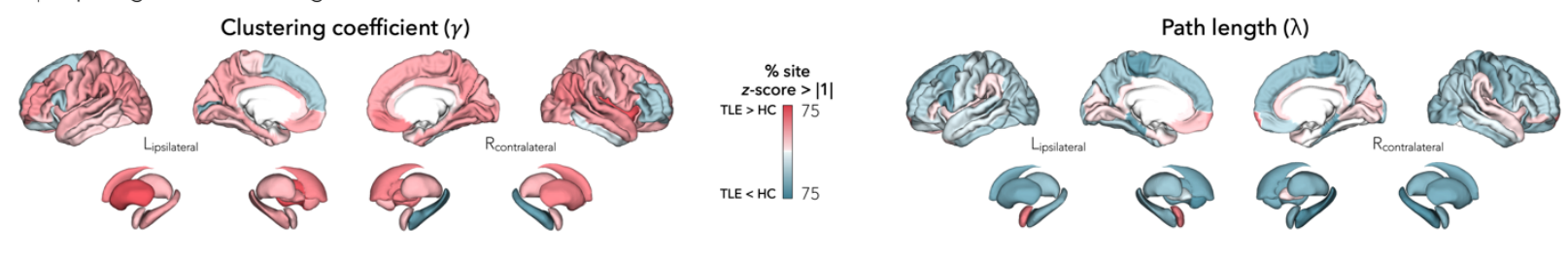

b | Topological network randomization in IGE across sites
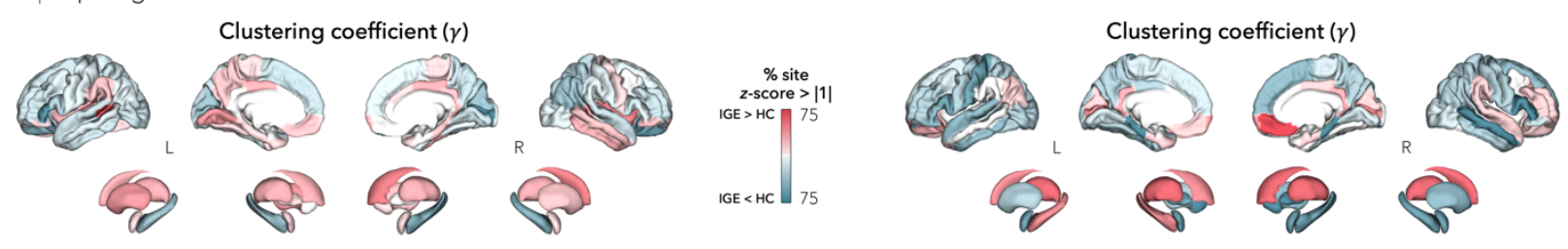

FIGURE S7. Site-specific structural covariance changes in TLE and IGE. Structural covariance and graph theoretical analyses were repeated in each site independently and yielded virtually identical results. (a) In TLE, increased clustering and path length were observed in bilateral orbitofrontal, temporal, and angular cortices, as well as ipsilateral amygdala, revealing a regularized, "lattice-like," subnetwork. (b) In IGE, widespread decreased in clustering and path length were observed in fronto-temporo-parietal regions, suggesting a randomized network configuration. 
bioRxiv preprint doi: https://doi.org/10.1101/2021.10.18.464713; this version posted October 19, 2021. The copyright holder for this preprint (which was not certified by peer review) is the author/funder, who has granted bioRxiv a license to display the preprint in perpetuity. It is made available under aCC-BY-NC-ND 4.0 International license.

a Stability of multivariate network findings across matrix thresholds
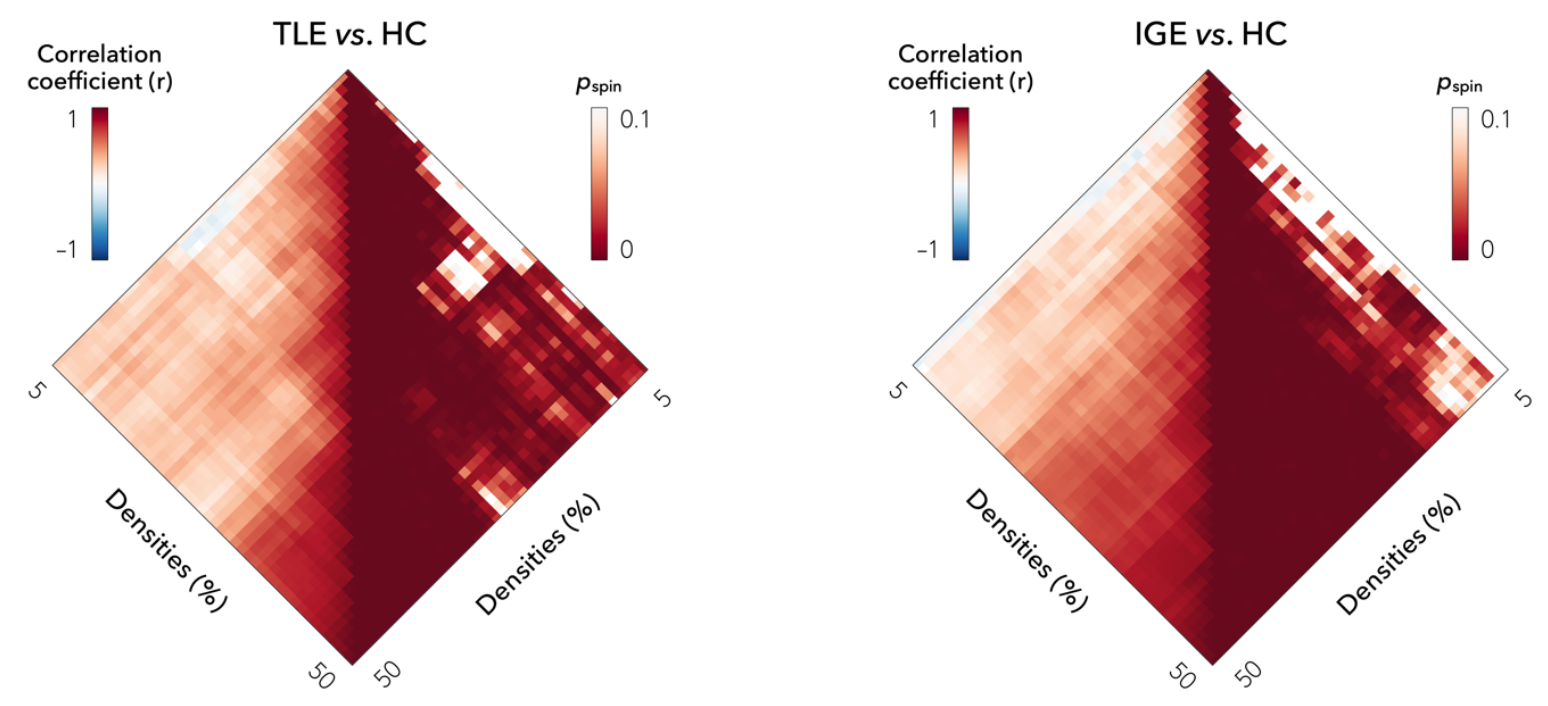

b | Stability of transcriptomic associations across matrix thresholds
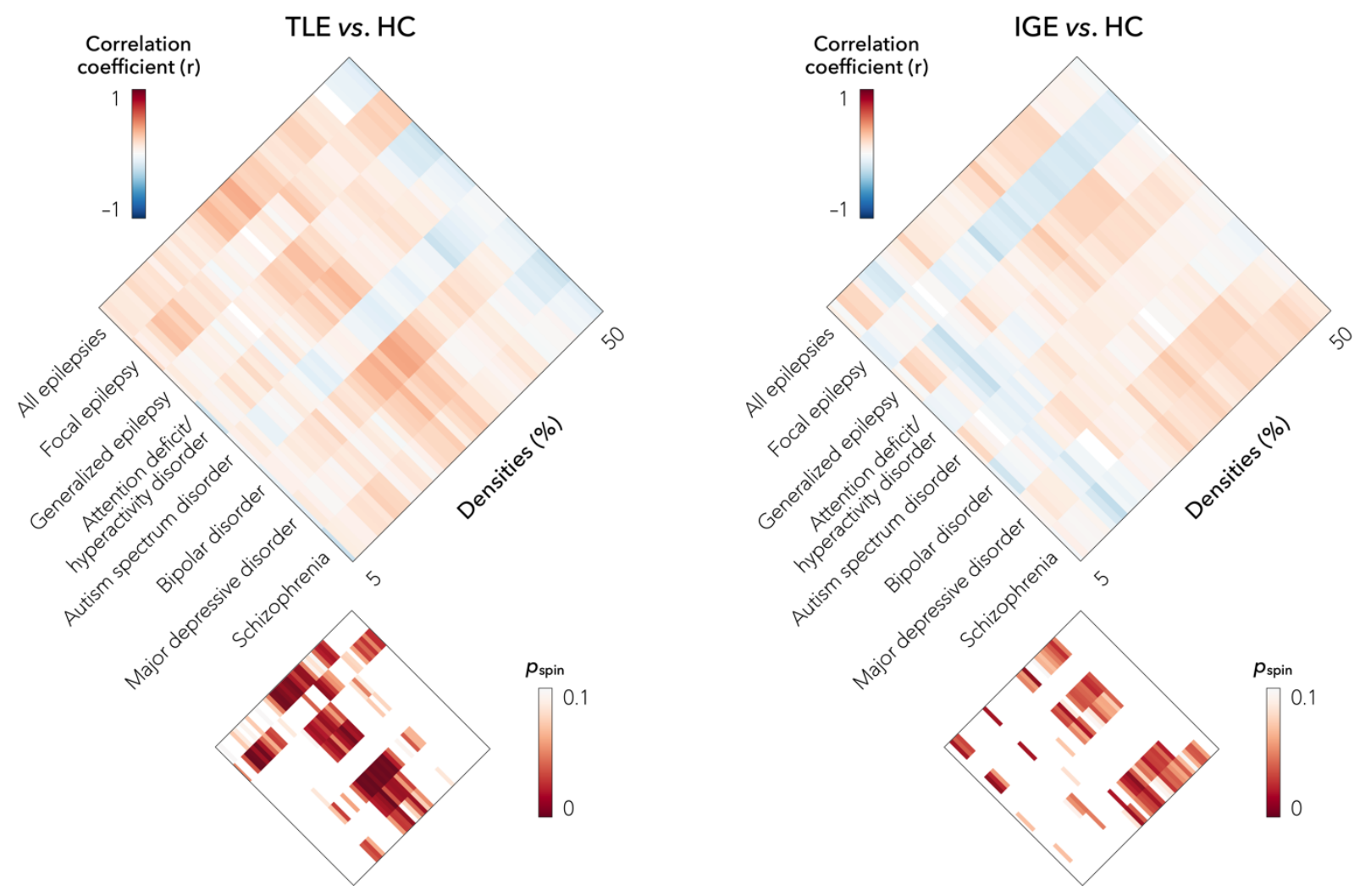

FIGURE S8. Reproducibility of findings across matrix thresholds. (a) Associations between multivariate topological changes (clustering and path length) across matrix thresholds. (b) Associations between diseaserelated gene co-expression maps and multivariate findings across matrix thresholds. 